\title{
Comparing Overall Success Rates of Cognitive-Behavioral Therapy, Eye Movement Desensitization and Reprocessing, Dialectical Behavior Therapy and Hypnosis
}

\author{
Edwin K. Yager \\ University of California at San Diego, La Jolla, CA, USA
}

\begin{abstract}
This study is a comparative analysis of the effectiveness of four psychotherapeutic treatment modalities: Cognitive-Behavioral Therapy (CBT), Eye Movement Desensitization and Reprocessing (EMDR), Dialectical Behavior Therapy (DBT), and Hypnotic methods (HYP). The aim of this study was to determine the independent effectiveness of the four treatment modalities by reviewing the available literature. A total of 26,724 studies were identified by ProQuest search and screened to eliminate studies that did not permit computation of the success rates of their use. Of that total, 648 studies included objective data in some form, with 207 studies qualifying for inclusion in the analysis. The "Success Rate" measure was used as the standard of measurement, and was obtained by comparing the pre-treatment scores with post-treatment scores as published in the qualifying studies. Analysis revealed the most effective treatment modality to be EMDR, with a success rate of $49.4 \%$. CBTwas the second most effective at $40.5 \%$, and the success rates of Hypnosis and DBT were $39.2 \%$ and $22.4 \%$ respectively. The clinical question in therapy should probably be, "How effective can I expect this method of treatment to be?" The findings of this study reveal CBT to be less effective than EMDR and equally effective as Hypnosis; and with DBT being significantly less effective, we are left with the implication that we must expand what we know.
\end{abstract}

Keywords: CBT, EMDR, DBT, hypnosis, effectiveness, efficacy, success rate

Although subjective opinions abound, objective evidence of the actual effectiveness of common psychotherapies is limited and at times produces contrary findings (Barth et al., 2013). Attempts are commonly made to value the benefit of one psychological treatment modality over another using controlled studies. However, considering the respective efficacy of modalities across studies is not common. For example, a meta-analysis by Gloaguen, Cottraux, Cucherat, and Ivy-Marie (1998) found CBT to be more effective than non-CBT approaches in depression treatment. In contrast, a meta-analysis by Leichsenring (2001) indicated no significant difference in efficacy between CBT and psychodynamic therapy for depression.

Rosenzweig (1936) suggested that all the various forms of psychological treatments are equally effective. He further proposed that it is not the technique which makes a difference, but other factors, including the simple presence of a safe space to discuss worries with a skilled and compassionate provider. Lambert (1992) tried to quantify this thesis and estimated that only $15 \%$ of client change occurred due to a specific technique.

Edwin K. Yager, Ph.D., Department of Psychiatry, School of Medicine, University of California at San Diego, La Jolla, CA, USA. 
He attributed the rest to motivation or severity of the problem (40\%), quality of the therapeutic relationship $(30 \%)$, and placebo effect (15\%). The actual benefit of psychotherapy in the treatment of mental health disorders still remains unclear.

Whereas Barth et al. (2013) compared the efficacy of seven interventions for depression alone; this current study was performed to examine the effectiveness of four treatment modalities of psychotherapy across various mental health problems and diagnoses. The modalities examined included Cognitive-Behavioral Therapy (CBT), Dialectical Behavior Therapy (DBT), Eye Movement Desensitization and Reprocessing (EMDR), and Hypnotic methods (HYP). Without direct comparisons between the effectiveness of psychological treatments across studies and across problem areas, it is difficult to establish the independent merits of each method. Understanding the merit of each treatment modality can further facilitate research into cost-benefit analysis and overall value of the modality.

\section{Method}

This study summarizes data contained in published studies of the methods cited in which the efficacy of each method was compared to one or more alternate methods. A significant percentage of all published papers on these methods, including both case and controlled studies, were identified. Following three succeeding filter steps used to identify the qualifying studies, the raw data were used to compute the success rates quoted. No discrimination was made between the types of problems or psychological disorders treated in the studies; the data represent the combined success rates of all problems reported in those studies.

\section{Success Rate}

The measure used in this study was "Success Rate," as opposed to Cohen's $d$ and/or other effect-size measures, because of the wide variation of the $n$ for the studies included. This "Success Rate" calculation produced the percentage change in reported mean scores between baseline and post-treatment measurements. Overall success rate for a treatment modality was obtained by averaging the success rates of all studies in which the modality was used. These success rates were then corrected for the wide variation in the $n$ of the studies.

The analysis is presented without claim of accuracy of the source data provided in the published literature; nevertheless, it reports a substantial portion of existing published studies. Moreover, it constitutes a challenge to the generally held beliefs and expectations regarding the effectiveness of the methods reviewed.

\section{Sources of the Data}

Four prevalent treatment methods were selected for inclusion and comparison: CBT, EMDR, DBT, and Hypnosis. The data were compiled from studies accessed via computer across three widely used electronic databases (EBSCOHOST's Psychology and Behavioral Sciences Collection, Academic Search Premier, and Science Direct). Initial computer search parameters retrieved only clinical trials published in a peer-reviewed journal from the databases. The exception to this search was for Hypnosis, where an additional online database, Taylor \& Francis, was searched for viable studies; examining only the first 4,000 results of the Taylor \& Francis database search.

\section{Screening the Data}

The initial computer search across the three databases yielded 26,724 studies in which one or more of this study's treatment methods existed. Employing three screenings successively, the data processor filtered out studies that did not meet the criteria for acceptance as defined in Table 1. 
Table 1

Acceptance Criteria for the Studies

(a) Studies must be clinical trials published in peer-reviewed academic journals.

(b) Studies must not be literature reviews.

(c) Studies must not be proposed trials.

(d) Studies must not be meta-analyses.

(e) Studies must be published in the English language.

(f) Studies must not use an adjunctive treatment method.

(g) Studies must report treatment time.

(h) Studies must report the number of patients.

(g) Studies must contain sufficient raw data to calculate a success rate.

(h) Studies must not be duplicates from the cross-database search.

The first (computerized) screen. The researcher examined 26,724 potential studies for basic criteria compliance, referred to as the first screen. This included inspecting the titles and abstracts of the studies for any violations of criteria (a) through (e), which reduced the number of usable studies to 3,529.

The second (manual) screen. All studies passing the first screen proceeded to a visual appraisal of the full contents of the published study, eliminating studies violating any of the criteria numbered (f) through (h), leaving 648 studies.

The third (manual) screen. The third and final screen included detailed, visual examinations of the remaining 648 studies, ensuring that each study met criteria (i) through (j). This left 198 studies, or $0.74 \%$ of the initial 26,724, which met all criteria for inclusion and therefore were included in this analysis.

Table 2

Summary of the Three Screenings

\begin{tabular}{lllll}
\hline & CBT & EMDR & DBT & HYP \\
\hline Studies identified in the initial search & 11,861 & 565 & 1,502 & 12,796 \\
Studies remaining after the first (computer) screen & 1,992 & 160 & 36 & 1,341 \\
Studies remaining after the second (manual) screen & 231 & 62 & 25 & 330 \\
Studies remaining after the third (manual) screen & 112 & 29 & 12 & 45 \\
\hline
\end{tabular}

\section{Analysis of Data}

The "Success Rate" measure was used as the standard of measurement and obtained by comparing the pre-treatment scores with post-treatment scores as published in the qualifying studies. Since the $n$ of the studies varied by a factor of ten or more, correction for this variance was made by multiplying the success rate of each study by the $n$ of the study, totaling these values and dividing by the total $n$ of the studies. Please see complete list of studies included in the analysis in the appendixes.

\section{Results}

The overall results of the analysis are summarized in Table 3. CBT studies, using 4,085 total subjects, resulted in a final success rate of $40.5 \%$. Data from 13 DBT studies, using 217 total subjects, resulted in a final success rate of $22.4 \%$. Data from 29 EMDR studies, using a total of 689 subjects, resulted in a final success rate of $49.4 \%$. Data from 42 HYP studies, using a total of 1,504 subjects, resulted in a final success rate of $39.2 \%$. 
The results of the analysis for CBT across problems treated are summarized in Table 4, those for DBT are summarized in Table 5, those for EMDR are summarized in Table 6, and those for HYP are summarized in Table 7.

Table 3

Overall Average Success Rates Across Treatment Modalities

\begin{tabular}{lllll}
\hline & CBT & EMDR & DBT & HYP \\
\hline Average number of treatments & 15.3 & 6.3 & 27.2 & 5.7 \\
Total studies included & 112 & 29 & 13 & 40 \\
Total number of subjects & 4085 & 689 & 217 & 1479 \\
Success rates & $40.5 \%$ & $49.4 \%$ & $22.4 \%$ & $39.8 \%$ \\
\hline
\end{tabular}

Table 4

CBT Average Success Rates by Problem Category

\begin{tabular}{lllll}
\hline Diagnosis & Studies & $n$ & Treatment hours & Success rate \\
\hline Addiction & 3 & 161 & 19.0 & 32.9 \\
Anxiety & 97 & 3,451 & 15.5 & 40.5 \\
Depression & 5 & 108 & 16.8 & 37.5 \\
Mood & 0 & & & 50.7 \\
Physical & 4 & 223 & 18.0 & \\
Sexual & 0 & & & 39.7 \\
Sleep & 4 & 120 & 7.9 & \\
Other & 0 & & & \\
\hline
\end{tabular}

Table 5

DBT Average Success Rates by Problem Category

\begin{tabular}{|c|c|c|c|c|}
\hline Diagnosis & Studies & $n$ & Treatment hours & Success rate \\
\hline \multicolumn{5}{|l|}{ Addiction } \\
\hline Anxiety & 3 & & 50.7 & 23.4 \\
\hline Depression & 1 & & & 23.5 \\
\hline \multicolumn{5}{|l|}{ Mood } \\
\hline Physical & 4 & & 20.8 & 3.2 \\
\hline \multicolumn{5}{|l|}{ Sexual } \\
\hline \multicolumn{5}{|l|}{ Sleep } \\
\hline Other & 4 & & 10.8 & 27.3 \\
\hline
\end{tabular}

Table 6

EMDR Average Success Rates by Problem Category

\begin{tabular}{|c|c|c|c|c|}
\hline Diagnosis & Studies & $\mathrm{n}$ & Treatment hours & Success rate \\
\hline \multicolumn{5}{|l|}{ Addiction } \\
\hline Anxiety & 30 & & 6.3 & 49.4 \\
\hline \multicolumn{5}{|l|}{ Depression } \\
\hline Mood & 1 & & 7.5 & 57.8 \\
\hline Physical & 1 & & 1.5 & 62.2 \\
\hline Sexual & 2 & & 9.0 & 46.5 \\
\hline \multicolumn{5}{|l|}{ Sleep } \\
\hline Other & & & & \\
\hline
\end{tabular}


Table 7

HYPAverage Success Rates by Problem Category

\begin{tabular}{|c|c|c|c|c|}
\hline Diagnosis & Studies & $\mathrm{n}$ & Treatment hours & Success rate \\
\hline \multicolumn{5}{|l|}{ Addiction } \\
\hline Anxiety & 4 & & 4.3 & 23.4 \\
\hline Depression & 1 & & 16 & 55.1 \\
\hline Mood & 1 & & 13 & 56.3 \\
\hline Physical & 25 & & 5.9 & 26.1 \\
\hline Sexual & 1 & & 9 & 25.0 \\
\hline Sleep & 2 & & 3.5 & 34.4 \\
\hline Other & 8 & & 3.5 & 46.0 \\
\hline
\end{tabular}

\section{Discussion}

The fact that objective data were found in less than $10 \%$ of the 26,724 studies brings into question the validity of frequently made claims of modality effectives. Casting further question on the validity of generally expected success rates, only 207 studies (less than $1 \%$ of the identified studies) contained objective data in a form that permitted computation of success rates. The unexpected reality of these numbers is considered significant in and of itself because it pinpoints the limitation to validating the methods.

These findings are concerning since even CBT, at a Success Rate of $40.5 \%$, has become widely accepted as the standard of care in the treatment of a wide variety of behavioral health disorders. CBT's conventionally understood, more elevated efficacy as the treatment of choice is undermined when compared to EMDR at $49.4 \%$ and HYP at $39.2 \%$.

\section{Limitations}

It is evident that comparing studies using different report measures can be problematic. The current analysis compared success rate (percentage change in scores between pre and post treatment) obtained by studies using different measures to evaluate reported symptoms. These measures include, but are not limited to: the Test Anxiety Inventory (TAI), Child Reaction Index (CRI), Revised Children's Manifest Anxiety Scale (RCMAS), and Beck Depression Inventory (BDI). Different measures use different scales and scoring systems. As such, simply comparing the percentage change between studies is an insufficient statistical analysis. However, the point of this paper is to give an overall impression of the efficacy of various treatment modalities, and the information from these findings is disappointing even though non-conclusive.

It is additionally limiting that within each study chosen for the analysis, several measures were occasionally reported and one might claim that choosing only one measure can lead to a selection bias. The measure chosen in such occasions was the most effective measure within that study.

This study does not intend to make claims dependent on statistical significance, but to shed light on the overall efficacy rates as reported by available studies in the field. A more robust meta-analysis that will compare scores on similar measures using a weighted system could support these findings with more consistent significant statistical results. In addition, it might be appropriate to consider additional treatment modalities if studies support results that show similar or higher success rates than those reported here.

\section{References}

Barth, J., Munder, T., Gerger, H., Nüesch, E., Trelle, S., Znoj, H, Jüni, P., \& Cuijpers. P. (2013). Comparative efficacy of seven psychotherapeutic interventions for patients with depression: A network meta-analysis. PLoS Med., 10(5), e1001454. PMCID: PMC3665892. 
Gloaguen, V., Cottraux, J., Cucherat, M., \& Ivy-Marie, B. (1998). A meta-analysis of the effects of cognitive therapy in depressed patients. Journal of Affective Disorders, 49, 59-72.

Lambert, M. J. (1992). Psychotherapy outcome research: Implications for integrative and eclectic therapists. In John C. Norcross and Marvin R. Goldfried's Handbook of Psychotherapy Integration (1st ed.) (pp. 94-129). New York: Basic Books. ISBN 0465028799. OCLC 25547822.

Leichsenring, F. (2001). Comparative effects of short-term psychodynamicpsychotherapy and cognitive-behavioral therapy in depression: A meta-analyticapproach. Clinical Psychology Review, 21, 401-419.

Rosenzweig, S. (1936). Some implicit common factors in diverse methods of psychotherapy. American Journal of Orthopsychiatry, 6, 412-415. doi:10.1111/j.1939-0025.1936.tb05248.x

Appendix A

Spread sheets and authors of the CBT method

Sheet $1-$ CBT raw data

\begin{tabular}{|c|c|c|c|c|c|c|c|c|c|c|}
\hline Author & Dx & Tx hrs & N1 & M1 & $\mathrm{Sd} 1$ & N2 & M2 & $\mathrm{Sd} 2$ & $\begin{array}{l}\% \\
\text { change }\end{array}$ & $\begin{array}{l}\text { Correction } \\
\text { weight for } n\end{array}$ \\
\hline ADDIS & PANIC D & 13.5 & 20 & 5.2 & 5.10 & 20 & 3.9 & 4.7 & 25 & 500.0 \\
\hline ARNTZ & GAD & 12 & 25 & 57.5 & 8.30 & 25 & 52.7 & 10.3 & 8.3 & 208.7 \\
\hline BARLOW & PANIC D & 15 & 15 & 5.5 & 0.90 & 15 & 2.6 & 1.4 & 52.7 & 790.9 \\
\hline BARLOW & GAD & 15 & 7 & 5.4 & 0.90 & 7 & 2.7 & 1.4 & 50.0 & 350.0 \\
\hline BOUCHARD & PANIC D & 22.5 & 14 & 1.5 & 1.55 & 14 & 0.13 & 0.2 & 91.3 & $1,278.7$ \\
\hline BRYANT & ASD & 7.5 & 33 & 27.1 & 7.46 & 33 & 16.58 & 12.5 & 38.9 & $1,282.5$ \\
\hline BUDNEY & CANNABIS & 14 & 30 & 7.9 & 4.00 & 26 & 5.1 & 4.7 & 35.4 & $1,063.3$ \\
\hline BURKE & AGORAPHOBIA & 30 & 12 & 29.3 & 7.71 & 12 & 15.16 & 10.5 & 48.2 & 578.1 \\
\hline BUTLER & GAD & 10.7 & 19 & 29.8 & 12.90 & 19 & 12.4 & 10.7 & 58.4 & $1,109.4$ \\
\hline CALEAR & ANX & & 530 & 8.9 & 6.41 & 484 & 7.86 & 7.0 & 11.5 & $6,087.8$ \\
\hline CARLBRING & PANIC D & 10 & 24 & 52.6 & 10.80 & 24 & 31.3 & 9.1 & 40.5 & 971.9 \\
\hline CHARD & PTSD & 35.5 & 36 & 65.5 & 26.39 & 36 & 9 & 11.0 & 86.3 & $3,105.0$ \\
\hline CHEN & ANX & 12 & 63 & 54.4 & 8.90 & 63 & 42.7 & 6.5 & 21.5 & $1,355.0$ \\
\hline CHOI & PANIC D & 24 & 20 & 61.4 & 11.04 & 20 & 42.42 & 8.7 & 30.9 & 618.7 \\
\hline CLARK & ANX & 5 & 7 & 7.4 & 2.60 & 7 & 5 & 1.8 & 32.4 & 227.0 \\
\hline COTTRAUX & OCD & 20 & 30 & 9.6 & 1.70 & 30 & 1.9 & 2.1 & 80.2 & $2,406.3$ \\
\hline CRASKE & AGORAPHOBIA & 24 & 34 & 5.9 & 4.80 & 34 & 1.19 & 2.1 & 79.9 & $2,715.4$ \\
\hline CRASKE & PANIC D & 11 & 24 & 5.9 & 0.80 & 24 & 2.4 & 1.7 & 59.3 & $1,423.7$ \\
\hline CRASKE & PANIC D & 22 & 20 & 19.0 & 12.00 & 20 & 0.9 & 1.5 & 95.3 & $1,905.3$ \\
\hline DANNON & PANIC D & 16 & 23 & 21.4 & 10.00 & 23 & 9.9 & 8.0 & 53.7 & $1,236.0$ \\
\hline DAVIDSON & SOCIAL PHOBIA & 14 & 60 & 39.2 & 10.40 & 48 & 20.6 & 9.9 & 47.4 & $2,846.9$ \\
\hline DeBEURS & PANIC D & 12 & 18 & 7.5 & 1.18 & 18 & 5.83 & 1.9 & 22.0 & 395.2 \\
\hline DeBEURS & PANIC D & 0 & 18 & 6.2 & 1.18 & 18 & 4.35 & 1.7 & 29.5 & 531.0 \\
\hline DeRUITER & PANIC D & 8 & 13 & 3.0 & 0.70 & 13 & 2.8 & 0.8 & 6.7 & 86.7 \\
\hline DUGAS & GAD & 28 & 25 & 6.4 & 1.19 & 25 & 3.4 & 1.8 & 46.5 & $1,163.5$ \\
\hline DURHAM & GAD & 18 & 14 & 1.8 & 0.70 & 14 & 0.8 & 0.8 & 55.6 & 777.8 \\
\hline DURHAM & GAD & 18 & 15 & 6.2 & 0.70 & 15 & 3 & 1.4 & 51.6 & 774.2 \\
\hline EHLERS & PTSD & 9.8 & 20 & 27.5 & 9.70 & 14 & 5 & 5.2 & 81.8 & $1,636.4$ \\
\hline FAIRBURN & PTSD & 8 & 25 & 70.9 & 16.20 & 25 & 37.5 & 30.4 & 47.1 & $1,177.7$ \\
\hline FAIRBURN & EATING D & 16.67 & 65 & 3.6 & 1.01 & 58 & 1.57 & 1.3 & 56.3 & $3,657.4$ \\
\hline FECYEAU & PTSD & 14.5 & 23 & 29.5 & 9.94 & 23 & 11.7 & 7.3 & 60.3 & $1,387.2$ \\
\hline FOA & PTSD & 13.5 & 14 & 24.5 & 6.62 & 14 & 11.07 & 4.0 & 54.8 & 766.9 \\
\hline FREESTON & OCD & 40.5 & 15 & 25.1 & 5.00 & 15 & 12.2 & 9.6 & 51.4 & 770.9 \\
\hline FROMMBERGER & PTSD & 12 & 10 & 70.5 & 7.20 & 10 & 34.8 & 15.0 & 50.6 & 506.4 \\
\hline
\end{tabular}




\begin{tabular}{|c|c|c|c|c|c|c|c|c|c|c|}
\hline GELERNTER & SOCIAL PHOBIA & 24 & 17 & 17.9 & 8.10 & 17 & 12.1 & 8.7 & 32.4 & 550.8 \\
\hline GERSONS & PTSD & 16 & 22 & 21.1 & 7.30 & 22 & 13.4 & 5.6 & 36.5 & 802.8 \\
\hline GRAZIANO & DEP & & 41 & 17.2 & 8.60 & 36 & 15.23 & 8.5 & 11.2 & 461.1 \\
\hline GREEN & MENOPAUSAL & 20 & 4 & 39.8 & 12.40 & 4 & 16.9 & 9.5 & 57.5 & 230.2 \\
\hline GREIST & OCD & 11 & 66 & 25.2 & 4.30 & 66 & 17.6 & 6.2 & 30.2 & $1,990.5$ \\
\hline GRUBER & SOCIAL PHOBIA & 30 & 5 & 52.7 & 8.00 & 5 & 40.7 & 11.8 & 22.8 & 113.9 \\
\hline GRUNES & OCD & 12 & 14 & 12.3 & 3.02 & 14 & 11.4 & 2.7 & 7.3 & 102.4 \\
\hline HEDMAN & PANIC D & & 570 & 10.5 & 4.90 & 404 & 5.3 & 4.8 & 49.5 & $28,228.6$ \\
\hline HERBERT & SAD & 12 & 14 & 128.3 & 37.39 & 14 & 74.8 & 38.0 & 41.7 & 583.5 \\
\hline HOPE & SOCIAL PHOBIA & 27 & 13 & 19.4 & 7.40 & 13 & 18.5 & 12.0 & 4.6 & 60.3 \\
\hline HSIEH & ANX & 12 & 9 & 11.9 & 3.33 & 9 & 8.75 & 3.5 & 26.4 & 237.7 \\
\hline JARNEFELT & INSOMNIA & 8 & 32 & 22.4 & 16.30 & 30 & 20.4 & 14.5 & 8.9 & 285.7 \\
\hline KAYROUZ & ANX & & 11 & 11.7 & 6.35 & 10 & 3.8 & 2.2 & 67.5 & 743.0 \\
\hline KENDALL & ANX & 16 & 54 & 48.8 & 20.13 & 54 & 36.93 & 22.0 & 24.3 & $1,311.0$ \\
\hline KENWRIGHT & OCD & 1.27 & 22 & 26.5 & 5.10 & 22 & 20.2 & 9.2 & 23.8 & 523.0 \\
\hline KOBAK & OCD & 12 & 28 & 22.8 & 3.68 & 28 & 16.32 & 7.0 & 28.5 & 797.5 \\
\hline $\mathrm{KOH}$ & PANIC D & 4.67 & 21 & 26.4 & 6.60 & 21 & 8.9 & 4.0 & 66.3 & $1,392.0$ \\
\hline KUBANY & PTSD & 14.5 & 46 & 72.9 & 18.40 & 45 & 15.8 & 14.4 & 78.3 & $3,603.0$ \\
\hline LADOUCEUR & GAD & 15.8 & 14 & 6.4 & 0.74 & 14 & 2.64 & 1.6 & 58.5 & 818.9 \\
\hline LANZA & SUBSTANCE D & 24 & 19 & 31.2 & 17.40 & 19 & 18 & 13.6 & 42.3 & 803.8 \\
\hline LEE & PTSD & 10.5 & 12 & 20.6 & 10.00 & 12 & 13.25 & 12.0 & 35.6 & 427.4 \\
\hline LIDDLE & SUBSTANCE D & & 112 & 28.5 & 17.36 & 112 & 19.75 & 18.2 & 30.6 & $3,430.4$ \\
\hline LILLIECREUTZ & PHOBIA & 2 & 30 & 52.8 & 6.52 & 30 & 45 & 6.3 & 14.8 & 443.2 \\
\hline LINDEN & GAD & 21.6 & 36 & 26.8 & 8.30 & 36 & 17.3 & 10.5 & 35.4 & $1,276.1$ \\
\hline LOERCH & PANIC D & 7.5 & 14 & 25.4 & 7.89 & 14 & 9.14 & 6.3 & 64.0 & 895.4 \\
\hline MARKS & PTSD & 10 & 18 & 3.2 & 0.80 & 18 & 1.6 & 1.2 & 50.0 & 900.0 \\
\hline MARKS & PANIC D & 6 & 29 & 7.3 & 1.00 & 29 & 3.6 & 1.3 & 50.7 & $1,469.9$ \\
\hline MCDONAGH & PTSD & 24.5 & 29 & 69.9 & 16.80 & 29 & 53.1 & 28.8 & 24.0 & 697.0 \\
\hline McEVOY & DEP & 20 & 38 & 26.9 & 8.90 & 38 & 16.5 & 11.3 & 38.7 & $1,469.1$ \\
\hline MCLEAN & OCD & 30 & 31 & 21.9 & 5.80 & 31 & 16.1 & 6.7 & 26.5 & 821.0 \\
\hline McMURCHIE & DEP & & 33 & 21.1 & 5.47 & 33 & 12.88 & 8.9 & 38.9 & $1,284.6$ \\
\hline MERSCH & SOCIAL PHOBIA & 16 & 20 & 5.2 & 1.12 & 20 & 2.87 & 1.3 & 44.3 & 885.4 \\
\hline NEUNER & PTSD & 6 & 17 & 25.2 & 7.40 & 15 & 19.1 & 11.7 & 24.2 & 411.5 \\
\hline NEWMAN & SOCIAL PHOBIA & 16 & 16 & 42.9 & 12.40 & 16 & 36.2 & 9.5 & 15.6 & 249.9 \\
\hline NEWMAN & PANIC D & 12 & 18 & 2.4 & 0.90 & 18 & 1.44 & 0.4 & 39.7 & 715.5 \\
\hline O'CONNOR & OCD & 20 & 15 & 25.5 & 7.10 & 15 & 13.3 & 8.6 & 47.8 & 717.6 \\
\hline OST & GAD & 12 & 18 & 23.9 & 6.45 & 18 & 11.39 & 5.9 & 52.4 & 943.6 \\
\hline OST & PANIC D & 15.19 & 26 & 5.2 & & 26 & 2.04 & & 60.7 & $1,578.0$ \\
\hline OST & PANIC D & 12 & 15 & 49.9 & 15.53 & 15 & 30.2 & 20.8 & 39.4 & 591.6 \\
\hline OTTO & SOCIAL PHOBIA & 30 & 20 & 4.9 & 0.80 & 15 & 3.5 & 1.3 & 28.6 & 571.4 \\
\hline PARKER & DEP & 10 & 11 & 16.1 & 5.60 & 11 & 10.6 & 7.4 & 34.2 & 375.8 \\
\hline PAUNOVIC & PTSD & 27 & 8 & 95.1 & 25.70 & 8 & 49 & 24.2 & 48.5 & 387.8 \\
\hline PAXLING & GAD & & 44 & 68.7 & 5.94 & 44 & 57.82 & 13.0 & 15.9 & 699.0 \\
\hline PETRY & GAMBLING & 8 & 70 & 8.7 & 3.90 & 70 & 2.9 & 3.6 & 66.7 & $4,666.7$ \\
\hline RESICK & PTSD & 12 & 62 & 74.8 & 18.77 & 62 & 39.08 & 31.1 & 47.7 & $2,959.0$ \\
\hline ROSS & PANIC D & 18 & 15 & 6.1 & 8.33 & 15 & 0.13 & 0.4 & 97.9 & $1,467.9$ \\
\hline RUSSELL & OCD & 14.4 & 23 & 24.8 & 3.70 & 23 & 17.8 & 8.4 & 28.2 & 649.2 \\
\hline RYBARCZYK & INSOMNIA & 16 & 46 & 49.8 & 38.60 & 46 & 22 & 17.8 & 55.8 & $2,567.9$ \\
\hline SALABERRIA & SOCIAL PHOBIA & 20 & 18 & 23.2 & 2.92 & 18 & 16.9 & 5.8 & 27.2 & 488.8 \\
\hline
\end{tabular}




\begin{tabular}{|c|c|c|c|c|c|c|c|c|c|c|}
\hline SANNIBALE & PTSD & 18 & 33 & 13.4 & 7.36 & 33 & 7.47 & 5.2 & 44.3 & $1,461.7$ \\
\hline SATO & INSOMNIA & 4.25 & 20 & 2.3 & 0.60 & 20 & 1.2 & 0.4 & 47.8 & 956.5 \\
\hline SCHEERS & FATIGUE & 16 & 84 & 48.4 & 7.10 & 84 & 29.3 & 14.0 & 39.5 & $3,314.9$ \\
\hline SCHNEIDER & PANIC D & & 43 & 7.0 & 1.20 & 33 & 4.7 & 2.0 & 32.9 & $1,412.9$ \\
\hline SCHOLING & SOCIAL PHOBIA & 16 & 30 & 26.9 & 6.40 & 30 & 16.1 & 7.9 & 40.1 & $1,204.5$ \\
\hline SCHOLING & SOCIAL PHOBIA & 8 & 15 & 24.0 & 7.50 & 15 & 12.5 & 6.9 & 47.9 & 718.8 \\
\hline SERFATY & EMOTIONAL & 8 & 19 & 46.3 & 21.60 & 19 & 26 & 21.0 & 43.8 & 833.0 \\
\hline SHARP & PANIC D & 12 & 30 & 21.3 & 4.10 & 30 & 7.1 & 7.5 & 66.7 & $2,000.0$ \\
\hline SHEAR & PANIC D & 15 & 24 & 4.0 & 4.10 & 20 & 1.2 & 3.2 & 70.0 & $1,680.0$ \\
\hline SPENCE & ANX & 10 & 22 & 6.0 & 1.02 & 22 & 2 & 2.3 & 66.7 & $1,466.7$ \\
\hline STANGIER & SOCIAL PHOBIA & 15 & 2 & 80.9 & 12.00 & 2 & 59.7 & 20.3 & 26.2 & 52.4 \\
\hline STANLEY & GAD & 21 & 31 & 5.1 & 0.26 & 31 & 1.83 & 1.9 & 64.2 & $1,989.8$ \\
\hline STANLEY & GAD & 15 & 29 & 60.1 & 9.47 & 29 & 51.6 & 10.2 & 14.1 & 410.1 \\
\hline STANLEY & GAD & 8 & 6 & 5.5 & 0.58 & 5 & 3.8 & 0.5 & 30.9 & 185.5 \\
\hline STEKETEE & HOARDING D & 12 & 23 & 63.4 & 14.95 & 23 & 53.73 & 18.5 & 15.3 & 351.4 \\
\hline TARRIER & PTSD & 16 & 33 & 77.8 & 14.95 & 33 & 50.82 & 24.0 & 34.6 & $1,143.3$ \\
\hline TAYLOR & SOCIAL PHOBIA & 28 & 32 & 174.8 & 18.00 & 32 & 154.2 & 23.6 & 11.8 & 377.1 \\
\hline TELCH & PANIC D & 18 & 34 & 4.2 & 9.47 & 34 & 0.18 & 1.3 & 95.7 & $3,253.6$ \\
\hline TROEUNG & DEP & 16 & 11 & 10.1 & 3.73 & 11 & 7.64 & 2.8 & 24.3 & 267.1 \\
\hline TURNER & SOCIAL PHOBIA & 30 & 21 & 122.3 & & 21 & 93.7 & & 23.4 & 491.1 \\
\hline VAN BALKOM & OCD & 12 & 25 & 28.0 & 7.00 & 19 & 12.5 & 9.3 & 55.4 & $1,383.9$ \\
\hline VAN OPPEN & OCD & 12 & 28 & 28.7 & 5.30 & 28 & 13.4 & 9.4 & 53.3 & $1,492.7$ \\
\hline VOGEL & OCD & 24 & 16 & 24.9 & 2.90 & 16 & 16.7 & 7.2 & 32.9 & 526.9 \\
\hline WALLER & BULIMIA & 19.2 & 70 & 4.5 & 6.51 & 70 & 1.84 & 5.1 & 58.7 & $4,105.6$ \\
\hline WETHERELL & GAD & 12 & 18 & 4.9 & 0.80 & 18 & 2.4 & 1.6 & 51.0 & 918.4 \\
\hline WHITTAL & OCD & 12 & 41 & 23.5 & 4.30 & 30 & 10.6 & 7.1 & 54.9 & $2,250.6$ \\
\hline YANG & SLEEP D & 3.5 & 22 & 48.0 & 11.79 & 22 & 27.18 & 7.6 & 43.4 & 954.3 \\
\hline \multirow[t]{2}{*}{ YOSHINAGA } & SAD & 21 & 15 & 91.8 & 23.50 & 15 & 51.7 & 27.8 & 43.7 & 655.2 \\
\hline & & 15.3 & 4,085 & & \multicolumn{5}{|c|}{ Corrected success rate average: $40.5 \%$} & $165,541.3$ \\
\hline
\end{tabular}

Sheet 2-Studies included

Addis, M. E, Hatgis, C., Cardemil, E. V., Jacob, K., Krasnow, A. D, \& Mansfield, A. (2006). Effectiveness of cognitive behavioral treatment of panic disorder versus treatment as usual in a managed care setting: Two yearfollow-up. Journal of Consulting and Clinical Psychology, 72(4), 625-635. doi:10.1037/0022-006x.72.4.625

Arntz, A. (2003). Cognitive therapy versus applied relaxation as treatment of generalized anxiety disorder. Behav Res Ther., 41(6), 633-646. doi:10.1016/s0005-7967(02)00045-1

Barlow, D. H., Craske, M. G., Cerny, J. A., \& Klosko, J. S. (1989). Behavioral treatment of panic disorder. Behav Ther., 20(2), 261-282. doi: 10.1016/s0005-7894(89)80073-5

Barlow, D. H., Rapee, R. M., \& Brown, T. A. (1992). Behavioral treatment of generalized anxiety disorder. Behav Ther., 23(4), 551-570. doi:10.1016/s0005-7894(05)80221-7

Bouchard, S., Gauthier, J., Laberge, B., French, D., Pelletier, M. H., \& Godbout, C. (1996). Exposure versus cognitive restructuring in the treatment of panic disorder with agoraphobia. Behav Res Ther., 34(3), 213-224. doi: 10.1016/0005-7967(95)00077-1

Bryant, R. A., Moulds, M. L., Guthrie, R. M., Dang, S. T., \& Nixon, R. D. (2003). Imaginal exposure alone and imaginal exposure with cognitive restructuring in treatment of posttraumatic stress disorder. J Consult Clin Psychol., 71(4), 706-712. doi: 10.1037/0022-006x.71.4.706

Bryant, R., Moulds, M., Guthrie, R., \& Nixon, R. (2005). The additive benefit of hypnosis and cognitive behavioral therapy in treating acute stress disorder. Journal of Consulting and Clinical Psychology, 73(2), 334-340. doi: 10.1037/0022-006x.73.2.334 
Budney, A. J., Moore, B. A., Rocha, H. L., \& Higgins, S. T. (2006). Clinical trial of abstinence-based vouchers and cognitive-behavioral therapy for cannabis dependence. Journal of Consulting and Clinical Psychology,74(2), 307-316. doi: 10.1037/0022-006x.4.2.307

Burke, M., Drummond, L. M., \& Johnston, D. W. (1997). Treatment choice for agoraphobic women: Exposure of cognitive behavior therapy? Br J Clin Psychol., 36(3), 409-420. doi: 10.1111/j.2044-8260.1997.tb01248.x

Butler, G., Fennell, M., Robson, P., \& Gelder, M. (1991). Comparison of behavior therapy and cognitive behavior therapy in the treatment of generalized anxiety disorder. Journal of Consulting and Clinical Psychology, 59(1), 167-175. doi: 10.1037//0022-006x.59.1.167

Calear, A. L., Christensen, H., Mackinnon, A., Griffiths, K. M., \& O’kearney, R. (2009). The YouthMood Project: A cluster randomized controlled trial of an online cognitive behavioral program with adolescents. Journal of Consulting and Clinical Psychology, 77(6), 1021-1032. doi:10.1037/a0017391

Carlbring, P., NilssonIhrfelt, E., Waara, J., Kollenstam, C., Buhrman, M., Kaldo, V., So“derberg, M., Ekselius, L., \& Andersson, G. (2005). Treatment of panic disorder: Live therapy versus selfhelp via Internet. Behav Res Ther., 43(1), 1321-1333. doi: 10.1016/j.brat.2004.10.002

Chard, K. M. (2005). An evaluation of cognitive processing therapy for the treatment of posttraumatic stress disorder related to childhood sexual abuse. J Consult Clin Psychol., 73(5), 965-971. doi: 10.1037/0022-006x.73.5.965

Chen, J., Chen, C., \& Zhi, S. (2014). Retrospective comparison of cognitive behavioral therapy and symptom-specific medication to treat anxiety and depression in throat cancer patients after laryngectomy. Shanghai Archives of Psychiatry, 26(2), 95-100. doi: $10.3969 / \mathrm{j}$

Choi, Y. H., Vincelli, F., Riva, G., Wiederhold, B. K., Lee, J. H., \& Park, K. H. (2005). Effects of group experiential cognitive therapy for the treatment of panic disorder with agoraphobia. Cyberpsychol Behav., 8(4), 387-393. doi:10.1089/cpb.2005.8.387

Clark, D. B., \& Agras, W. S. (1991). The assessment and treatment of performance anxiety in musicians. Am J Psychiatry., 148(5), 598-605. doi:10.1176/ajp.148.5.598

Cottraux, J., Note, I., Yao, S. N., Lafont, S., Note, B., Mollard, E., Bouvard, M., Sauteraud, A., Bourgeois, M., \& Dartigues, J. F. (2001). A randomized controlled trial of cognitive therapy versus intensive behavior therapy in obsessive compulsive disorder. Psychother Psychosom., 70(6), 288-297. doi: 10.1159/000056269

Craske, M. G., DeCola, J. P., Sachs, A. D., \& Pontillo, D. C. (2003). Panic control treatment of agoraphobia. J Anxiety Disord., 17(3), 321-333. doi: 10.1016/s0887-6185(02)00203-7

Craske, M. G., Lang, A. J., Aikins, D., \& Mystkowski, J. L. (2005). Cognitive behavioral therapy for nocturnal panic. Behav Ther., 36(1), 43-54. doi: 10.1016/s0005-7894(05)80053-x

Craske, M. G., Rowe, M., Lewin, M., \& Noriega, D. R. (1997). Interoceptive exposure versus breathing retraining within cognitive behavioral therapy for panic disorder with agoraphobia. $\mathrm{Br} J$ Clin Psychol., 36(1), 85-99. doi: 10.1111/j.2044-8260.1997.tb01233.x

Craske, M. G., Street, L., Barlow, D. H. (1989). Instructions to focus upon or distract from internal cues during exposure treatment of agoraphobic avoidance. Behav Res Ther., 27(6), 663-672. doi: 10.1016/0005-7967(89)90150-2

Dannon, P. N., GonUsishkin, M., Gelbert, A., Lowengrub, K., \& Grunhaus, L. (2004). Cognitive behavioral group therapy in panic disorder patients: The efficacy of CBGT versus drug treatment. Ann Clin Psychiatry, 16(1), 41-46. doi: $10.1080 / 10401230490281609$

Davidson, J. R. T., Foa, E. B., Huppert, J. D., Keefe, F. J., Franklin, M. E., Compton, J. S., Zhao, N., Connor, K. M., Lynch, T. R., \& Gadde, K. M. (2004). Fluoxetine, comprehensive cognitive behavioral therapy and placebo in generalized social phobia. Arch Gen Psychiatry, 61(10), 1005-1013. doi: 10.1001/archpsyc.61.10.1005

deBeurs, E., van Balkom, A. J. L. M., Lange, A., Koele, P., \& van Dyck, R. (1995). Treatment of panic disorder with agoraphobia: Comparison of fluvoxamine, placebo, psychological panic management combined with exposure and of exposure in vivo alone. Am J Psychiatry, 152(5), 683-691. doi: 10.1176/ajp.152.5.683

deBeurs, E., van Balkom, A. J. L. M., Van Dyck, R., \& Lange, A. (1999). Long-term outcome of pharmacological and psychological treatment of panic disorder with agoraphobia: A 2-year naturalistic follow-up. Acta Psychiatr Scand., 99(1), 59-67. doi:10.1111/j.1600-0447.1999.tb05385.x

De Ruiter, C., Rijken, H., Garssen, B., \& Kraaimaat, F. (1989). Breathing retraining, exposure and a combination of both, in the treatment of panic disorder with agoraphobia. Behav Res Ther., 27(6), 647-655. doi: 10.1016/0005-7967(89)90148-4 
Dugas, M. J., Ladouceur, R., Leger, E., Freeston, M. H., Langlois, F., Provencher, M. D., \& Boisvert, J. M. (2003). Group cognitive behavioral therapy for generalized anxiety disorder: Treatment outcome and long-term followup. $J$ Consult Clin Psychol., 71(4), 821-825. doi: 10.1037/0022-006x.71.4.821

Durham, R. C., Chambers, J. A., MacDonald, R. R., Power, K. G., \& Major, K. (2003). Does cognitive behavioral therapy influence the long-term outcome of generalized anxiety disorder? An 8-14 year follow-up of 2 clinical trials. Psychol Med., 33(3), 499-509. doi: 10.1017/s0033291702007079

Durham, R. C., Fisher, P. L., Trevling, L. R., Hau, C. M., Richard, K., \& Stewart, J. B. (1999). One year follow-up of cognitive therapy, analytic psychotherapy and anxiety management training for generalized anxiety disorder: Symptom change, medication usage and attitudes to treatment. Behav Cogn Psychother., 27(1), 19-35.

Durham, R. C., Murphy, T., Allan, T., Richard, K., Treliving, L. R., \& Fenton, G. W. (1994). Cognitive therapy, analytic psychotherapy and anxiety management training for generalized anxiety disorder. Br J Psychiatry., 165(3), 315-323. doi: 10.1192/bjp.165.3.315

Ehlers, A., Clark, D. M., Hackmann, A., McManus, F., \& Fennell, M. (2005). Cognitive therapy for posttraumatic stress disorder: Development and evaluation. Behav Res Ther., 43(4), 413-431. doi: 10.1016/j.brat.2004.03.006

Fairburn, C., Bailey Straebler, S., Basden, S., Doll, H., Jones, R., Murphy, R., \& Cooper, Z. (2015). A transdiagnostic comparison of enhanced cognitive behaviour therapy (CBTE) and interpersonal psychotherapy in the treatment of eating disorders. Behaviour Research and Therapy, 70, 64-71. doi:10.1016

Fecteau, G., \& Nicki, R. (1999). Cognitive behavioral treatment of post traumatic stress disorder after motor vehicle accident. Behav Cogn Psychother., 27(3), 201-214.

Foa, E. B., Rothbaum, B. O., Riggs, D. S., \& Murdock, T. B. (1991). Treatment of post-traumatic stress disorder in rape victims: A comparison between cognitive-behavioral procedures and counseling. J Consult Clin Psychol., 59(5), 715-723.

Freeston, M. H., Ladouceur, R., Gagnon, F., Thibodeau, N., Rheaume, J., \& Letarte, H. (1997). Cognitive behavioral treatment of obsessive thoughts: A controlled study. J Consult Clin Psychol., 65(3), 405-413. doi: 10.1037//0022-006x.65.3.405

Frommberger, U., Stieglitz, R., Nyberg, E., Richter, H., Novelli-Fischer, U., Angenendt, J., Zaninelli, R., \& Berger, M. (2004). Comparison between paroxetine and behavior therapy in patients with posttraumatic stress disorder (PTSD): A pilot study. Int J Psychiatry Clin Pract., 8(1), 19-23. doi: 10.1080/13651500310004803

Gelernter, C. S., Uhde, T. W., Cimbolic, P., Arnkoff, D. B., Vittone, B. J., \& Tancer, M. E. (1991). Cognitive behavioral and pharmacological treatments of social phobia. Arch Gen Psychiatry, 48(10), 938-945. doi:10.1001/archpsyc.1991.01810340070009

Gersons, B. P. R., Carlier, I. V. E., Lamberts, R. D., \& van Der Kolk, B. A. (2000). Randomized clinical trial of brief eclectic psychotherapy for police officers with posttraumatic stress disorder. J Trauma Stress, 13(2), 333-347. doi: 10.1023/A:1007793803627

Graziano, F., Calandri, E., Borghi, M., \& Bonino, S. (2014). The effects of a group-based cognitive behavioral therapy on people with multiple sclerosis: A randomized controlled trial. Clinical Rehabilitation, 28(3), 264274. doi:10.1177/0269215513501525

Greist, J. H., Marks, I. M., Baer, L., Kobak, K. A., Wenzel, K. W., \& Hirsch, J. (2002). Behavior therapy for obsessive compulsive disorder guided by a computer or by a clinician compared with relaxation as a control. J Clin Psychiatry, 63(2), 138-145. doi: 10.4088/jcp.v63n0209

Green, S., Haber, E., McCabe, R., \& Soares, C. (2013). Cognitive behavioral group treatment for menopausal symptoms: A pilot study. Archive of Womens Mental Health, 16(4), 325-332. doi:10.1007/s00737-013-0339-x

Grober, K., Moran, P. J., Roth, W. T., \& Taylor, C. B. (2001). Computer-assisted cognitive behavioral group therapy for social phobia. Behav Ther., 32(1), 155- 165. doi: 10.1016/S0005-7894(01)80050-2

Grunes, M. S., Neziroglu, F., \& McKay, D. (2001). Family involvement in the behavioral treatment of obsessive compulsive disorder. Behav Ther., 32(4), 803-820. doi: 10.1016/S0005-7894(01)80022-8

Hedman, E., Ljotsson, B., Ruck, C., Bergstrom, J., Andersson, G., Kaldo, V., \& Blom, K. (2013). Effectiveness of internet-based cognitive behaviour therapy for panic disorder in routine psychiatric care. Acta Psychiatrica Scandinavica, 128(6), 457-467. doi:10.1111/acps.12079

Herbert, J. D., Rheingold, A. A., Gaudiano, B. A., \& Myers, V. H. (2004). Standard versus extended cognitive behavior therapy for social anxiety disorder: A randomized controlled trial. Behav Cogn Psychother., 32(2), 131-147. doi: $10.1017 / \mathrm{S} 1352465804001171$ 
Hope, D. A., Heimberg, R. G., \& Bruch, M. A. (1995). Dismantling cognitive behavioral group therapy for social phobia. Behav Res Ther., 33(6), 637-650. doi: 10.1016/0005-7967(95)00013-N

Hsieh, M., Ponsford, J., Wong, D., Schonberger, M., Taffe, J., \& Mckay, A. (2012). Motivational interviewing and cognitive behaviour therapy for anxiety following traumatic brain injury: A pilot randomised controlled trial. Neuropsychological Rehabilitation, 22 (4), 585-608. doi:10.1080/09602011.2012.678860

Jarnefelt, H., Lagerstedt, R., Kajaste, S., Sallinen, M., Savolainen, A., \& Hublin, C. (2012). Cognitive behavior therapy for chronic insomnia in occupational health services. Journal of Occupational Rehabilitation, 22, 51121. doi:10.1007/s10926-012-9365-1

Kayrouz, R., Dear, B., Johnston, L., Gandy, M., Fogliati, V., Sheehan, J., \& Titov, N. (2015). A feasibility open trial of guided Internet-delivered cognitive behavioural therapy for anxiety and depression amongst Arab Australians. Internet Interventions, 2, 32-38. doi:10.1016/j.invent.2014.12.001

Kendall, P., Hudson, J., Gosch, E., Flannery, Schroeder, E., \& Suveg, C. (2008). Cognitive-behavioral therapy for anxiety disordered youth: A randomized clinical trial evaluating child and family modalities. Journal of Consulting and Clinical Psychology, 76 (2), 282-297. doi:10.1037/0022-006x.76.2.282

Kenwright, M., Marks, I., Graham, C., Franses, A., \& Mataix-Cols, D. (2005). Brief scheduled phone support from a clinician to enhance computer-aided self-help for obsessive-compulsive disorder: Randomized controlled trial. J Clin Psychol., 61(12), 1499-1508. doi: 10.1002/jclp.20204

Kobak, K., Greist, R., Jacobi, D., LevyMack, H., \& Greist, J. (2015). Computer-assisted cognitive behavior therapy for obsessive-compulsive disorder: A randomized trial on the impact of lay vs. professional coaching. Annals of General Psychiatry, 14, 10. doi:10.1186/s12991-015-0048-0

Koh, K. B., \& Lee, Y. (2004). Reduced anxiety level by therapeutic interventions and cell-mediated immunity in panic disorder patients. Psychother Psychosom, 73(5), 286-292. doi: 1159/000078845

Kubany, E. S., Hill, E. E., Owens, J. A., Spencer, C. I., McCaig, M. A., Tremayne, K. J., \& Williams, P. L. (2004). Cognitive trauma therapy for battered women with PTSD (CTTBW). J Consult Clin Psychol., 72(1), 3-18. doi: 10.1037/0022-006X.72.1.3

Ladouceur, R., Dugas, M. J., Freeston, M. H., Leger, E., Gagnon, F., \& Thibodeau, N. (2000). Efficacy of a cognitive behavioral treatment of generalized anxiety disorder: Evaluation in a controlled clinical trial. J Consult Clin Psychol., 68(6), 957-964. doi: 10.1016/j.jagp.2016.06.006

Lanza, P., Garcia, P., Lamelas, F., \& Gonzalez-Menendez, A. (2014). Acceptance and commitment therapy versus cognitive behavioral therapy in the treatment of substance use disorder with incarcerated women. Journal of Clinical Psychology, 70(7), 644-657. doi:10.1002/jclp.22060

Lee, C., Gavriel, H., Drummond, P., Richards, J., \& Greenwald, R. (2002). Treatment of PTSD: Stress inoculation training with prolonged exposure compared with EMDR. J Clin Psychol., 58(9), 1071-1089. doi: 10.1002/jclp.10039

Liddle, H., Dakof, G., Turner, R., \& Henderson, C. (2008). Treating adolescent drug abuse: A randomized trial comparing multidimensional family therapy and cognitive behavior therapy. Addiction, 103(10), 1660-1670. doi: 10.1111/j.1360-0443.2008.02274.x

Lilliecreutz, C., Josefsson, A., \& Sydsjo, G. (2009). An open trial with cognitive behavioral therapy for blood and injection phobia in pregnant women group intervention program. Archive of Womens Mental Health, 13, $259-265$. doi:10.1007/s00737-009-0126-x

Linden, M., Zubraegel, D., Baer, T., Franke, U., \& Schlattmann, P. (2005). Efficacy of cognitive behavior therapy in generalized anxiety disorders. Psychother Psychosom, 74(1), 36-42. Doi:10.1159/000082025

Loerch, B., GrafMorgenstern, M., Hautzinger, M., Schlegel, S., Hain, C., \& Sandmann, J. (1999). Randomized placebo-controlled trial of moclobemide, cognitive-behavioral therapy and their combination in panic disorder with agoraphobia. $\mathrm{Br} J$ Psychiatry, 174, 205-212. doi: 10.13109/zptm.2016.62.3.252

McEvoy, P., \& Nathan, P. (2007). Effectiveness of cognitive behavior therapy for diagnostically heterogeneous groups: A benchmarking study. Journal of Consulting and Clinical Psychology, 75(2), 344-350. doi:10.1037/0022-006x.75.2.344

McMurchie, W., Macleod, F., Power, K., Laidlaw, K., \& Prentice, N. (2013). Computerised cognitive behavioral therapy for depression and anxiety with older people: A pilot study to examine patient acceptability and treatment outcome. International Journal of Geriatric Psychiatry, 28, 1147-1156. doi:10.1002/gps.3935

Marks, I., Lovell, K,. Noshirvani, H., Livanou, M., \& Thrasher, S. (1998). Treatment of posttraumatic stress disorder by exposure and /or cognitive restructuring. Arch Gen Psychiatry, 5(4), 317-325. doi: 10.1001/archpsyc.55.4.317 
Marks, I. M., Kenwright, M., McDonough, M., Whittaker, M., \& Mataix-Cols, D. (2004). Saving clinicians' time by delegating routine aspects of therapy to a computer: A randomized controlled trial in phobia/panic disorder. Psychol Med., 34(1), 9-17. doi: $10.1017 / \mathrm{S} 003329170300878 \mathrm{X}$

McDonagh, A., Friedman, M., McHugo, G., Ford, J., Sengupta, A., Mueser, K., Demment, C. C., Fournier, D., Schnurr, P. P., \& Descamps, M. (2005). Randomized trial of cognitive-behavioral therapy for chronic posttraumatic stress disorder in adult female survivors of childhood sexual abuse. J Consult Clin Psychol., 73(3), 515-552. doi: 10.1037/0022-006X.73.3.515

McLean, P. D., Whittal, M.L., Thordarson, D. S., Taylor, S., Sochting, I., Koch, W. J., \& Paterson, R. (2001). Cognitive versus behavior therapy in the group treatment of obsessive-compulsive disorder. J Consult Clin Psychol., 69(2), 205-214. doi: 10.1037//0022-006x.69.2.205

Mersch, P. P. (1995). The treatment of social phobia: The differential effectiveness of exposure in vivo and an integration of exposure in vivo, rational emotive therapy and social skills training. Behav Res Ther., 33(3), 259-269. doi: 10.1016/0005-7967(94)00038-L

Neuner, F., Schauer, M., Klaschik, C., Karunakara, U., \& Elbert, T. (2004). A comparison of narrative exposure therapy, supportive counseling, and psychoeducation for treating posttraumatic stress disorder in an African refugee settlement. $J$ Consult Clin Psychol., 72(4), 579-587. doi: 10.1037/0022-006X.72.4.579

Newman, M. G., Hofmann, S. G., Trabert, W., Roth, W. T., \& Taylor, C. B. (1994). Does behavioral treatment of social phobia lead to cognitive changes? Behav Ther., 25(3), 503-517. doi: 10.1016/S0005-7894(05)80160-1

Newman, M. G., Kenardy, J., Herman, S., \& Taylor, C. B. (1997). Comparison of palmtop computer assisted brief cognitive-behavioral treatment to cognitive-behavioral treatment of panic disorder. J Consult Clin Psychol., 65(1), 178-183. doi: 10.1037/0022-006X.65.1.178

O'Connor, K. P., Aardema, F., Bouthillier, D., Fournier, S., Guay, S., Robillard, S., Pelissier, M. C., Landry, P., Todorov, C., Tremblay, M., \& Pitre, D. (2005). Evaluation of an inference-based approach to treating obsessive-compulsive disorder. Cogn Behav Ther., 34(3), 148-163. doi: 10.1080/16506070510041211

Ost, L. G., \& Breiholtz, E. (2000). Applied relaxation versus cognitive therapy in the treatment of generalized anxiety disorder. Behav Res Ther., 38(8), 777-790. doi: 10.1016/S0005-7967(99)00095-9

Ost, L.G., Thulin U., \& Ramnero, J. (2004). Cognitive behavior therapy versus exposure in vivo in the treatment of panic disorder with agoraphobia. Behav Res Ther. 42(10), 1105-1127. doi: 10.1016/j.brat.2003.07.004

Ost, L.G., \&Westling, B.E. (1995). Applied relaxation versus cognitive behavior therapy in the treatment of panic disorder. Behav Res Ther. 33(2), 145-158. doi: 10.1016/005-7967(94)E0026-F

Otto, M. W., Pollack, M. H., Gould, R. A., Worthington, III J. J., McArdle, E. T., \& Rosenbaum, J. F. (2000). A comparison of the efficacy of clonazepam and cognitive-behavioral group therapy for the treatment of social phobia. $J$ Anxiety Disord., 14(4), 345-358. doi: 10.1016/S0887-6185(00)00027-X

Parker, G., Blanch, B., Paterson, A., HadziPavlovic, D., Sheppard, E., Manicavasagar, V., \& Friend, P. (2013). The superiority of antidepressant medication to cognitive behavior therapy in melancholic depressed patients: A 12-week single-blind randomized study. Acta Psychiatrica Scandinavica, 128(4), 271-281. doi: 10.1111/acps.12049

Paunovic, N., \& Ost, L. (2001). Cognitive-behavior therapy versus exposure therapy in the treatment of PTSD in refugees. Behav Res Ther., 39(10), 1183-1197. doi: 10.1016/S0005-7967(00)00093-0

Paxling, B., Almlov, J., Dahlin, M., Carlbring, P., Breitholtz, E., Eriksson, T., \& Andersson, G. (2011). Guided internet-delivered cognitive behavior therapy for generalized anxiety disorder: A randomized controlled trial. Cognitive Behaiour Therapy, 40(3), 159-173. doi:10.1080/16506073.2011.576699

Petry, N., Ammerman, Y., Bohl, J., Doersch, A., Gay, H., Kadden, R., \& Molina, C. (2006). Cognitive-behavioral therapy for pathological gamblers. Journal of Consulting and Clinical Psychology, 74(3), 555-567. doi: 10.1037/0022-006X.74.3.555

Resick, P. A., Nishith, P., Weaver, T. L., Astin, M. C., \& Feuer, C. A. (2002). A comparison of cognitive-processing therapy with prolonged exposure and a waiting condition for the treatment of chronic posttraumatic stress disorder in female rape victims. J Consult Clin Psychol., 70(4), 867-879. doi: 10.1037/0022-006X.70.4.867

Ross, C. J., Davis, T. M., \& MacDonald, G. F. (2005). Cognitive behavioral treatment combined with asthma education for adults with asthma and coexisting panic disorder. Clin Nurs Res., 14(2), 131-157. doi: 10.1177/1054773804273863

Rybarczyk, B., Stepanski, E., Fogg, L., Lopez, M., Barry, P., \& Davis, A. (2005). A placebo-controlled test of cognitive-behavioral therapy for comorbid insomnia in older adults. Journal of Consulting and Clinical Psychology, 73(6), 1164-1174. doi: 10.1037/0022-006X.73.6.1164 
Salaberria, K., \& Echeburua, E. (1998). Long-term outcome of cognitive therapy's contribution to self-exposure in vivo to the treatment of generalized social phobia. Behav Mod., 22(3), 262-284. doi: 10.1177/01454455980223003

Sannibale, C., Teesson, M., Creamer, M., Sitharthan, T., Bryant, R., Sutherland, K., \& Bostock-Matusko, D. (2013). Randomized controlled trial of cognitive behaviour therapy for comorbid posttraumatic stress disorder and alcohol use disorders. Addiction, 108(8), 1397-1410. doi:10.1111/add.12167

Sato, M., Yumadera, W., Matsushima, M., Itoh, H., \& Nakayama, K. (2010). Clinical efficacy of individual cognitive behavior therapy for psychophysiological insomnia in 20 outpatients. Psychiatry and Clinical Neurosciences, 64(2), 187-195. doi: 10.1111/j.1440-1819.2009.02060.x

Scheeres, K., Wensing, M., Knoop, H., \& Bleijenberg, G. (2008). Implementing cognitive behavioral therapy for chronic fatigue syndrome in a mental health center: A benchmarking evaluation. Journal of Consulting and Clinical Psychology, 76(1), 163-171. doi:10.1037/0022-006x.76.1.16

Schneider, A. J., Mataix-Cols, D., Marks, I. M., \& Bachofen, M. (2005). Internet guided self help with or without exposure therapy for phobic and panic disorders: A randomized controlled trial, 74(3), 154-164. doi: 10.1159/000084000

Scholing, A., \& Emmelkamp, P. M. (1993a). Exposure with and without cognitive therapy for generalized social phobia: Effects of individual and group treatment. Behav Res Ther., 31(7), 667-681. doi: 10.1016/0005-7967(93)90120-J

Scholing, A., \& Emmelkamp, P. M. (1993b). Cognitive and behavioral treatments of fear of blushing, sweating or trembling. Behav Res Ther., 31(2), 155-170. doi: 10.1016/0005-7967(93)90067-5

Serfaty, M., Wilkinson, S., Freeman, C., Mannix, K., \& King, M. (2012). The ToT sudy: Helping with touch or talk (ToT): A pilot randomised controlled trial to examine the clinical effectiveness of aromatherapy massage versus cognitive behaviour therapy for emotional distress in patients in cancer/palliative care. PsychoOncology, 21(5), 563-569. doi: 10.1002/pon.1921

Sharp, D., Power, K. G., \& Swanson, V. (2004). A comparison of the efficacy and acceptability of group versus individual cognitive behavioral therapy in the treatment of panic disorder and agoraphobia in primary care. Clin Psychol Psychother., 11(2), 73-82. doi: 10.1002/cpp.393

Shear, K., Pilkonis, P. A., Cloitre, M., \& Leon, A. C. (1994). Cognitive behavioral treatment compared with non-prescriptive treatment of panic disorder. Arch Gen Psychiatry, 51(5), 395-401. doi: 10.1001/archpsyc.1994.03950050055006

Spence, S., Donovan, C., March, S., Gamble, A., Anderson, R., Prosser, S., \& Kenardy, J. (2011). A randomized controlled trial of online versus clinic-based CBT for adolescent anxiety. Journal of Consulting and Clinical Psychology, 79(5), 629-642.

Stangier, U., Heidenreich, T., Peitz M., Lauterbach, W., \& Clark, D. M. (2003). Cognitive therapy for social phobia: Individual versus group treatment. Behav Res Ther., 41(9), 991-1007. doi: 10.1016/S0005-7967(02)00176-6

Stanley, M. A., Beck, J. G., \& Glassco, J. D. (1996). Treatment of generalized anxiety in older adults: A preliminary comparison of cognitive-behavioral and supportive approaches. Behav Ther., 2(4), 565-581. doi: 10.1016/S0005-7894(96)80044-X

Stanley, M. A., Beck, J.G., Novy, D. M., Averill, P. M., Swann, A. C., Diefenbach, G. J., \& Hopko, D. R. (2003a). Cognitive behavioral treatment of late-life GAD. J Consult Clin Psychol., 71(2), 309-319. doi: 10.1037/0022-006X.71.2.309

Stanley, M. A., Hopko, D. R., Diefenbach, G. J., Bourland, S. L., Rodriguez, H., \& Wagener, P. (2003b) Cognitive-behavior therapy for late-life generalized anxiety disorder in primary care: Preliminary findings. Am J Geriatr Psychiatry, 11(1), 92-96. doi: 10.1097/00019442-200301000-00012

Steketee, G., Frost, R., Tolin, D., Rasmussen, J., \& Brown, T. (2010). Waitlist-controlled trial of cognitive behavior therapy for hoarding disorder. Depression and Anxiety, 27(5), 476-484. doi:10.1002/da.20673

Tarrier, N., Pilgrim H., Sommerfield, C., Faragher, B., Reynolds, M., Graham, E., \& Barrowclough, C. (1999). A randomized trial of cognitive therapy and imaginal exposure in the treatment of chronic posttraumatic stress disorder. J Consult Clin Psychol., 67(1), 13-18. doi: 10.1037/0022-006X.67.1.13

Taylor, S., Woody, S., Koch, W. J., McLean, P., Paterson, R. J., \& Anderson, K. W. (1997). Cognitive restructuring in the treatment of social phobia. Behav Mod., 21(4), 487-511. doi: 10.1177/01454455970214006

Telch, M. J., Lucas, J. A., Schmidt, N. B., Hanna, H. H., Jaimez, T. L., \& Lucas, R. A. (1993). Group cognitive-behavioral treatment of panic disorder. Behav Res Ther., 3(3), 279-287. doi: 10.1016/0005-7967(93)90026-Q

Troeung, L., Egan, S., \& Gasson, N. (2014). A waitlist-controlled trial of group cognitive behavioral therapy for depression and anxiety in Parkinson's disease. BMC Psychiatry, 14, 19. doi: 10.1186/1471-244X-14-19

Turner, S. M., Beidel, D. C., \& Jacob, R. G. (1994). Social phobia: A comparison of behavior therapy and Atenolol. J Consult Clin Psychol., 62(2), 350-358. doi: 10.1037//0022-006X.62.2.350 
van Balkom, A. J., de Haan, E., van Oppen, P., Spinhoven, P., Hoogduin, K. A., \& van Dyck, R. (1998). Cognitive and behavioral therapies alone versus in combination with fluvoxamine in the treatment of obsessive compulsive disorder. J Nerv Ment Dis., 186(8), 492-499. doi: 10.1097/00005053-199808000-00007

van Oppen, P., De Hann, E., van Balkom, A. J. L. M., Spinhoven, P., Hoogduin, K., \& van Dyck, R. (1995). Cognitive therapy and exposure in vivo in the treatment of obsessive compulsive disorder. Behav Res Ther., 33(4), 379-390. doi: 10.1016/0005-7967(94)00052-L

Vincelli, F., Anolli L., Bouchard, S., Wiederhold, B. K., Zurloni, V., \& Riva, G. (2003). Experiential cognitive therapy in the treatment of panic disorders with agoraphobia: A controlled study. Cyberpsychol Behav., 6(3), 321-328. doi: 10.1089/109493103322011632

Vogel, P. A., Stiles, T. C., \& Gotestam, K. G. (2004). Adding cognitive therapy elements to exposure therapy for obsessive compulsive disorder: A controlled study. Behav Cogn Psychother., 32(3), 275-290. doi: 10.1017/S1352465804001353

Waller, G., Gray, E., Hinrichsen, H., Mountford, V., Lawson, R., \& Patient, E. (2014). Cognitive-behavioral therapy for bulimia nervosa and atypical bulimic nervosa: Effectiveness in clinical settings. International Journal of Eating Disorders, 47(1), 13-17. doi: 10.1002/eat.22181

Wetherell, J. L., Gatz, M., \& Craske, M. G. (2003). Treatment of generalized anxiety disorder in older adults. J Consult Clin Psychol., 71(1), 31-40. doi: 10.1037/0022-006X.71.1.31

Whittal, M. L., Thordarson, D., \& McLean, P. D. (2005). Treatment of obsessive compulsive disorder: Cognitive behavior therapy versus exposure and response prevention. Behav Res Ther., 43(12), 1559-1576. doi: 10.1016/j.brat.2004.11.012

Yang, H., \& Petrini, M. (2012). Effect of cognitive behavior therapy on sleep disorder in Parkinson's disease in China: A pilot study. Nursing and Health Sciences, 14(4), 458-463. doi: 10.1111/j.1442-2018.2012.00711.x

Yoshinaga, N., Ohshima, F., Matsuki, S., Tanaka, M., Kobayashi, T., Ibuki, H., \& Shimizu, E. (2013). A preliminary study of individual cognitive behavior therapy for social anxiety disorder in Japanese clinical settings: A single-arm, uncontrolled trial. BMC Research Notes, 6,74. doi: 10.1186/1756-0500-6-74

\section{Appendix B}

Spread sheets and authors of the EMDR method

Sheet $1-$ EMDR raw data

\begin{tabular}{|c|c|c|c|c|c|c|c|c|c|c|}
\hline Author & Dx & Tx hrs & N1 & M1 & SD1 & $\mathrm{N} 2$ & M2 & SD2 & $\%$ change & \\
\hline ADRUIZ & PTSD & 5.0 & 124 & 7.2 & & 124 & 2.2 & & 69.6 & $8,628.3$ \\
\hline BALCOM & SHAME & 7.5 & 9 & 45.6 & & 9 & 19.2 & & 57.8 & 520.2 \\
\hline BLOOMGARDEN & EATING D & 1.5 & 43 & 6.1 & 3.1 & 43 & 2.3 & 5.3 & 62.2 & $2,676.0$ \\
\hline СНЕМТОВ & PTSD & 4.0 & 32 & 36.5 & 11.6 & 32 & 16.5 & 13.0 & 54.9 & $1,757.6$ \\
\hline EDMOND & SEXUAL & 6.0 & 6 & 16.3 & 7.2 & 6 & 8.6 & 6.7 & 47.2 & 283.4 \\
\hline ENRIGHT & ANXIETY & 2.0 & 35 & 65.5 & 8.4 & 35 & 51.8 & 15.4 & 21.0 & 734.7 \\
\hline FESKE & PANIC D & 5.0 & 15 & 45.3 & 25.1 & 15 & 27.1 & 26.4 & 40.2 & 602.6 \\
\hline GOLDSTEIN & ANX & 6.0 & 18 & 8.8 & 3.0 & 18 & 6.7 & 3.5 & 24.1 & 434.2 \\
\hline GRAINGER & PTSD & 2.0 & 29 & 7.7 & 1.6 & 29 & 1.9 & 2.1 & 74.9 & $2,171.2$ \\
\hline GREENWALD & PTSD & 3.0 & 15 & 6.2 & 1.6 & 15 & 2.2 & 2.3 & 64.5 & 967.0 \\
\hline HEBER & PTSD & 4.0 & 1 & 47.0 & & 1 & 23.0 & & 51.1 & 51.1 \\
\hline HEIDE & PTSD & 11.0 & 5 & 12.8 & 1.8 & 5 & 10.8 & 4.7 & 15.6 & 78.1 \\
\hline INOUE & PTSD & 20.0 & 1 & 25.0 & & 1 & 21.0 & & 16.0 & 16.0 \\
\hline IRONSON & PTSD & 6.0 & 10 & 26.6 & 11.6 & 10 & 9.1 & 11.2 & 65.8 & 657.6 \\
\hline JABERGHADERI & SEXUAL & 12.0 & 7 & 34.9 & 5.8 & 7 & 18.9 & 7.9 & 45.8 & 320.9 \\
\hline JONGH & PHOBIA & 2.0 & 1 & 10.0 & & 1 & 0.0 & & 100.0 & 100.0 \\
\hline KONUK & PTSD & 8.0 & 41 & 34.3 & 8.0 & 41 & 5.4 & 4.8 & 84.3 & $3,457.9$ \\
\hline LEE & PTSD & 4.6 & 12 & 16.8 & 7.8 & 12 & 8.2 & 5.7 & 51.0 & 611.8 \\
\hline MARCUS & PTSD & 5.8 & 62 & 17.9 & 16.5 & 42 & 12.3 & 14.6 & 31.2 & $1,932.7$ \\
\hline MAXWELL & ANX & 6.0 & 1 & 32.0 & & 1 & 6.0 & & 81.3 & 81.3 \\
\hline MURIS & PHOBIA & 2.0 & 9 & 10.3 & 1.5 & 9 & 8.8 & 2.9 & 15.0 & 135.0 \\
\hline
\end{tabular}




\begin{tabular}{|c|c|c|c|c|c|c|c|c|c|c|}
\hline NAZARI & OCD & 8.0 & 30 & 24.8 & 5.4 & 30 & 13.6 & 5.5 & 45.2 & $1,356.8$ \\
\hline NIJDAM & PTSD & 6.5 & 70 & 7.8 & 39.4 & 70 & 6.3 & 19.9 & 19.2 & $1,346.2$ \\
\hline ORAS & PTSD & 2.5 & 13 & 61.8 & 11.9 & 13 & 33.5 & 19.4 & 45.8 & 595.3 \\
\hline POWER & PTSD & 10.0 & 27 & 35.1 & 4.4 & 27 & 24.0 & 8.7 & 31.6 & 853.8 \\
\hline ROOS & PTSD & 4.0 & 26 & 30.5 & 11.5 & 26 & 17.7 & 9.6 & 42.0 & $1,091.1$ \\
\hline ROTHBAUM & PTSD & 9.0 & 20 & 26.0 & 7.1 & 20 & 10.7 & 11.5 & 58.8 & $1,175.3$ \\
\hline TAYLOR & PTSD & 8.0 & 15 & 26.4 & 10.0 & 15 & 16.4 & 9.1 & 37.9 & 568.2 \\
\hline \multirow[t]{2}{*}{ WADAA } & PTSD & 12.0 & 12 & 38.9 & 7.0 & 12 & 12.3 & 6.2 & 68.4 & 820.6 \\
\hline & & 6.3 & 689 & \multicolumn{6}{|c|}{ Corrected success rate average: $49.4 \%$} & $34,025.4$ \\
\hline
\end{tabular}

\section{Sheet 2-EMDR studies included}

Aduriz, M., Bluthgen, C., \& Knopfler, C. (2009). Helping child flood victims using group EMDR intervention in Argentina: Treatment outcomes and gender differences. Argentine Society of Psychotrauma, 16(2), 138-153.

Balcom, D., Call, E., \& Pearlman, D. (2000). Eye movement desensitization and reprocessing treatment of internalized shame. Traumatology, 6(2), 69-83.

Bloomgarden, A., \& Calogero, R. (2008). A randomized experimental test of the efficacy of EMDR treatment on negative body image in eating disorder inpatients. Eating Disorders, 16, 418-427.

Chemtob, C., Nakashima, J., \& Carlson, J. (2002). Brief treatment for elementary School children With disaster-related Posttraumatic Stress Disorder: A field Study. Journal of Clinical Psychology, 58(1), 99-112.

Edmond, T., \& Rubin, A. (2016). Assessing the long-term rffects Of EMDR: Results from an 18-month follow-up study with adult female survivors Of CSA. Journal of Child Sexual Abuse, 13, 69-86.

Enright, M., Baldo, T., \& Wykes, S. (2000). The efficacy of eye movement desensitization and reprocessing therapy technique in the treatment of test anxiety of college students. Journal of College Counseling, 3(1), 36-48.

Feske, U., \& Goldstein, A. (1997). Eye movement desensitization and reprocessing treatment for panic disorder: A controlled outcome and partial dismantling study. Journal of Consulting and Clinical Psychology, 65(6), 1026-1035.

Goldstein, A., Beurs, E., Chambless, D., \& Wilson, K. (2000). EMDR for panic disorder with agoraphobia: Comparison with waiting list and credible attention-placebo control conditions. Journal of Consulting and Clinical Psychology, 68(6), 947-956.

Grainger, R., Levin, C., Allen-Byrd, L., Doctor, R., \& Lee, H. (1997). An empirical evaluation of Eye Movement Desensitization and Reprocessing (EMDR) with survivors of a natural disaster. Journal of Traumatic Stress, 10(4), 665-671.

Greenwald, R., Mcclintock, S., \& Bailey, T. (2013). A Controlled Comparison of Eye Movement Desensitization \& Reprocessing and Progressive Counting. Journal of Aggression, Maltreatment \& Trauma, 22, 981-996.

Heber, R., Kellner, M., \& Yehuda, R. (2002). Salivary cortisol levels and the cortisol response to dexamethasone before and after EMDR: A case report. Journal of Clinical Psychology, 58(12), 1521-1530.

Heide, F., Mooren, T., Kleijn, W., Jongh, A., \& Kleber, R. (2011). EMDR versus stabilization in traumatized asylum seekers and refugees: Results of a pilot study. European Journal of Psychotraumatology, 2, 5811-5821.

Inoue, N. (2009). Evaluation of an EMDR Treatment outcome using the Rorschach, the TAT, and the IES-R. Rorschachiana, 30(2), 180-218.

Ironson, G., Freund, B., Strauss, J., \& Williams, J. (2002). Comparison of two treatments for traumatic stress: A community-based study of EMDR and prolonged exposure. Journal of Clinical Psychology, 58(1), 113-128.

Jaberghaderi, N., Greenwald, R., Rubin, A., Zand, S., \& Dolatabadi, S. (2004). A comparison of CBT And EMDR For sexually-abused Iranian girls. Clinical Psychology \&Psychotherapy, 11(5), 358-368.

Jongh, A., \& Broeke, E. (1998). Treatment of choking phobia by targeting traumatic memories with EMDR: A case study. Clinical Psychology \& Psychotherapy, 5, 264-269.

Konuk, E., Knipe, J., Eke, I., Yuksek, H., Yurtsever, A., \& Ostep, S. (2006). The effects of eye movement desensitization and reprocessing (EMDR) therapy on posttraumatic stress disorder in survivors of the 1999 Marmara, Turkey, earthquake. International Journal of Stress Management, 13, 291-308.

Lee, C., Gavriel, H., Drummond, P., Richards, J., \& Greenwald, R. (2002). Treatment of PTSD: Stress inoculation training with prolonged exposure compared to EMDR. Journal of Clinical Psychology, 58, 1071-1089.

Marcus, S., Marquis, P., \& Sakai, C. (2004). Three- and 6-Month Follow-Up of EMDR Treatment of PTSD in an HMO Setting. International Journal of Stress Management, 11, 195-208. 
Maxwell, J. (2003). The imprint of childhood physical and emotional abuse: A case study on the use of EMDR to address anxiety and a lack of self-esteem. Journal of Family Violence,18(5), 281-293.

Muris, P., Merckelbach, H., Holdrinet, I., \& Sijsenaar, M. (1998). Treating phobic children: Effects of EMDR versus exposure. Journal of Consulting and Clinical Psychology, 66, 193-198.

Nazari, H., Momeni, N., Jariani, M., \& Tarrahi, M. (2011). Comparison of eye movement desensitization and reprocessing with Citalopram in treatment of obsessive-compulsive disorder. International Journal of Psychiatry in Clinical Practice,15, 270-274.

Nijdam, M., Gersons, B., Reitsma, J., Jongh, A., \& Olff, M. (2012). Brief eclectic psychotherapy v. eye movement desensitization and reprocessing therapy for post-traumatic stress disorder: Randomized controlled trial. The British Journal of Psychiatry, 200, 224-231.

Oras, R., Ezpeleta, S., \& Ahmad, A. (2004). Treatment of traumatized refugee children with eye movement desensitization and reprocessing in a psychodynamic context. Nordic Journal of Psychiatry, 58(3), 199-203.

Power, K., Mcgoldrick, T., Brown, K., Buchanan, R., Sharp, D., Swanson, V., \& Karatzias, A. (2002). A controlled comparison of eye movement desensitization and reprocessing versus exposure plus cognitive restructuring versus waiting list in the treatment of post-traumatic stress disorder. Clinical Psychology \& Psychotherapy, 9, 299-318.

Roos, C., Greenwald, R., Hollander-Gijsman, M., Noorthoorn, E., Buuren, S., \& Jongh, A. (2011). A randomized comparison of cognitive behavioral therapy (CBT) and eye movement desensitization and reprocessing (EMDR) in disaster-exposed children. European Journal of Psychotraumatology, 2, 1-11.

Rothbaum, B., Astin, M., \& Marsteller, F. (2005). Prolonged exposure versus eye movement desensitization and reprocessing (EMDR) for PTSD rape victims. Journal of Traumatic Stress, 18(6), 607-616.

Taylor, S., Thordarson, D. S., Maxfield, L., Fedoroff, I. C., Lovell, K., \& Ogrodniczuk, J. (2003). Comparative efficacy, speed, and adverse effects of three PTSD treatments: Exposure therapy, EMDR, and relaxation training. Journal of Consulting and Clinical Psychology, 71(2), 330-338. doi:10.1037/0022-006x.71.2.330

Wadaa, N., Zaharim, N., \& Alqashan, H. (2010). The Use of EMDR in Treatment of Traumatized Iraqi Children. Digest of Middle East Studies, 19, 26-36.

\section{Appendix C}

Spread sheets and authors of the DBT method

Sheet $1-D B T$ raw data

\begin{tabular}{lllllllllll}
\hline Author & Dx & Tx & \multirow{2}{*}{ N1 } & M1 & SD1 & N2 & M2 & SD2 & \% change \\
\hline CHEN & BINGE-EATING & 23.0 & 8 & 2.38 & 3.16 & 8 & 1.29 & 1.6 & 45.8 & 366.4 \\
COURBASSON & SUBSTANCE D & 48.0 & 14 & 22.4 & 18.2 & 8 & 1.5 & 3 & 93.3 & $1,306.3$ \\
FELIU-SOLER & BPD & 20.0 & 18 & 4.72 & 1.88 & 18 & 4.61 & 2.03 & 2.3 & 41.9 \\
HILL & BINGE-EATING D & 12.0 & 18 & 3.61 & 1.16 & 18 & 2.48 & 1.39 & 31.3 & 563.4 \\
KEUTHEN & TRICHOTILLOMANIA & 15.0 & 10 & 18.5 & - & 10 & 7 & - & 62.2 & 621.6 \\
KLEIN & BINGE-EATING D & 40.0 & 5 & 4.7 & 3.11 & 5 & 3.75 & 1.5 & 20.2 & 101.1 \\
LYNCH & ANOREXIA NERVOSA & 8.0 & 34 & 14.69 & 1.49 & 34 & 18.26 & 2.18 & -24.3 & -826.3 \\
MEANEY-TAVARES & DEP AND ANX & 16.0 & 17 & 31.94 & 12.18 & 17 & 19.06 & 11.13 & 40.3 & 685.5 \\
NEE ( STUDY 1) & BPD & 4.0 & 1 & 21.07 & - & 1 & 8.68 & - & 58.8 & 58.8 \\
NEE ( STUDY 2) & BPD & 4.0 & 1 & 19.46 & - & 1 & 0.54 & - & 97.2 & 97.2 \\
RITSHCEL & DEP AND ANX & 70.0 & 55 & 32.47 & 12.78 & 42 & 26.34 & 15.08 & 18.9 & $1,038.3$ \\
STIEL & PTSD & 66.0 & 29 & 2.13 & 0.4 & 25 & 1.66 & 0.69 & 22.1 & 639.9 \\
WASSER & DEPRESSION & & 7 & 2.43 & 1.56 & 7 & 1.86 & 1.62 & 23.5 & 164.2 \\
& & 27.2 & 217 & & Corrected success rate average: $39.2 \%$ & $4,858.4$ \\
\hline
\end{tabular}

\section{Sheet 2-DBT studies included}

Chen, E., Matthews, L., Allen, C., Kuo, J., \& Linehan, M. (2008). Dialectical behavior therapy for clients with binge-eating disorder or bulimia nervosa and borderline personality disorder. International Journal of Eating Disorders, 41(6), 505-512. 
Courbasson, C., Nishikawa, Y., \& Dixon, L. (2012). Outcome of dialectical behavior therapy for concurrent eating and substance use disorders. Clinical Psychology \& Psychotherapy, 19(5), 434-449. doi: 10.1002/cpp.748

Feliu-Soler, A., Pascual, J., Borràs, X., Portella, M., Martín-Blanco, A., Armario, A., . . Soler, J. (2013). Effects of dialectical behavior therapy-mindfulness training on emotional reactivity in borderline personality disorder: Preliminary results. Clinical Psychology \& Psychotherapy, 21(4), 363-370. doi: 10.1002/cpp.1837

Hill, D., Craighead, L., \& Safer, D. (2011). Appetite-focused dialectical behavior therapy for the treatment of binge eating with purging: A preliminary trial. International Journal of Eating Disorders, 44, 249-261.

Keuthen, N., Rothbaum, B., Welch, S., Taylor, C., Falkenstein, M., Heekin, M., . . J Jenike, M. (2010). Pilot trial of dialectical behavior therapy-enhanced habit reversal for trichotillomania. Depression and Anxiety, 27, 953-959.

Klein, A., Skinner, J., \& Hawley, K. (2012). Adapted group-based dialectical behavior therapy for binge eating in a practicing clinic: Clinical outcomes and attrition. European Eating Disorders Review, 20(3), 148-153. doi: 10.1002/erv.2165

Lynch, T., Gray, K., Hempel, R., Titley, M., Chen, E., \& O’Mahen, H. (2013). Radically open-dialectical behavior therapy for adult anorexia nervosa: Feasibility and outcomes from an inpatient program. BMC Psychiatry, 13, 293.

Meaney-Tavares, R., \& Hasking, P. (2013). Coping and regulating emotions: A pilot study of a modified dialectical behavior therapy group delivered in a college counseling service. Journal of American College Health, 61(5), 303-309.

Nee, C., \& Farman, S. (2007). Dialectical behavior therapy as a treatment for borderline personality disorder in prisons: Three illustrative case studies. Journal of Forensic Psychiatry \& Psychology, 18(2), 160-180.

Ritschel, L., Cheavens, J., \& Nelson, J. (2012). Dialectical Behavior Therapy in an Intensive Outpatient Program With a Mixed-Diagnostic Sample. Journal of Clinical Psychology, 68, 221-235.

Steil, R., Dyer, A., Priebe, K., Kleindienst, N., \& Bohus, M. (2011). Dialectical behavior therapy for posttraumatic stress disorder related to childhood sexual abuse: A pilot study of an intensive residential treatment program. Journal of Traumatic Stress, 24, $102-106$.

Wasser, T., Tyler, R., McIlhaney, K., Taplin, R., \& Henderson, L. (2008). Effectiveness of dialectical behavior therapy (DBT) versus standard therapeutic milieu (STM) in a Cohort of Adolescents Receiving Residential Treatment. Best Practices in Mental Health,4(2), 114-125.

\section{Appendix D}

Spread sheets and authors of the HYP method

Sheet $1-$ HYP raw data

\begin{tabular}{|c|c|c|c|c|c|c|c|c|c|c|}
\hline Author & Dx & Tx hrs & N1 & M1 & SD1 & N2 & M2 & SD2 & $\%$ change & \\
\hline ABRAMOWITZ & INSOMNIA & 4.0 & 17 & 35.9 & 2.3 & 17 & 27.5 & 1.8 & 23.4 & 397.8 \\
\hline ANBAR & EATING D & 7.0 & 1 & 7 & & 1 & 2 & & 71.4 & 71.4 \\
\hline ALLADIN & DEPRESSION & 16.0 & 82 & 39 & & 82 & 17.5 & & 55.1 & $4,520.5$ \\
\hline ASKAY & PAIN & 1.0 & 27 & 67.8 & 22.2 & 27 & 55.6 & 28.9 & 18.0 & 485.8 \\
\hline BARKLEY & SMOKING & 7.0 & 8 & 16.6 & & 8 & 8.1 & & 51.2 & 409.6 \\
\hline BOLOCOFSKY & WEIGHT & 9.0 & 57 & 70.2 & & 57 & 66.2 & & 5.7 & 324.8 \\
\hline BOUTIN & $\begin{array}{l}\text { TEST } \\
\text { ANXIETY }\end{array}$ & 8.0 & 1 & 17 & & 1 & 5 & & 70.6 & 70.6 \\
\hline CARMODY & SMOKING & 2.0 & 145 & 100 & & 145 & 0.45 & 5.7 & 99.6 & $14,434.8$ \\
\hline DEIKER & COMPULSION & 4.0 & 1 & 19 & & 1 & 1 & & 94.7 & 94.7 \\
\hline DICKSON- sp & CESSATION & 1.0 & 116 & 100 & & 107 & 0.67 & & 99.3 & $11,522.7$ \\
\hline ELKINS & PAIN & 5.0 & 13 & 91.31 & & 13 & 25.38 & & 72.2 & 938.7 \\
\hline ELKINS & PAIN & 2.7 & 21 & 100 & & 21 & 19 & & 81.0 & $1,701.0$ \\
\hline ELKINS & LIBEDO & 9.0 & 1 & 8 & & 1 & 6 & & 25.0 & 25.0 \\
\hline ENEA & PAIN & 1.0 & 15 & 15 & 3.74 & 15 & 6.13 & 3.13 & 59.1 & 887.0 \\
\hline GALOVSKI & IBS & 12.0 & 11 & 13.55 & 8.77 & 11 & 10 & 7.62 & 26.2 & 288.2 \\
\hline GALOVSKI & IBS & 6.0 & 1 & 68 & & 1 & 52 & & 23.5 & 23.5 \\
\hline GAY & $\begin{array}{l}\text { HYPERTENSI } \\
\text { ON }\end{array}$ & 8.0 & 15 & 88.7 & 7.3 & 15 & 80 & 5.3 & 9.8 & 147.1 \\
\hline GRONDAHL & $\begin{array}{l}\text { CHRONIC } \\
\text { PAIN }\end{array}$ & 10.0 & 12 & 51.5 & & 12 & 41.6 & & 19.2 & 230.7 \\
\hline
\end{tabular}




\begin{tabular}{|c|c|c|c|c|c|c|c|c|c|c|}
\hline HAWKINS & NAUSEA & 1.0 & 10 & 12.3 & 3.3 & 10 & 8.3 & 1.5 & 32.5 & 325.2 \\
\hline HAWKINS & SLEEP D & 3.0 & 6 & 3.5 & & 6 & 1.2 & & 65.7 & 394.3 \\
\hline HORTON-HAUs & ARTHRITUS & 10.0 & 26 & 62.5 & & 26 & 27.5 & & 56.0 & $1,456.0$ \\
\hline JENSEN & PAIN & 10.0 & 8 & 3.21 & 1.76 & 8 & 1.32 & 1.28 & 58.9 & 471.0 \\
\hline JENSEN & PAIN & 10.0 & 26 & 4.64 & 1.95 & 26 & 3.78 & 2.35 & 18.5 & 481.9 \\
\hline JENSEN & CANCER PAIN & 4.0 & 5 & 5.13 & 0.77 & 5 & 4 & 0.47 & 22.0 & 110.1 \\
\hline JENSEN & PAIN & 10.0 & 23 & 5.99 & 1.833 & 23 & 5.09 & 1.92 & 15.0 & 345.6 \\
\hline JOHNSON & $\begin{array}{l}\text { LEARN } \\
\text { DISABIL }\end{array}$ & 3.0 & 15 & 14.8 & 4.9 & 15 & 15.53 & 5.11 & -4.9 & -74.0 \\
\hline LINDFORS & IBS & 12.0 & 83 & 27.5 & 7.2 & 83 & 24.6 & 7.8 & 10.5 & 875.3 \\
\hline LIOSSI & PAIN & 2.0 & 10 & 4 & & 10 & 2 & & 50.0 & 500.0 \\
\hline LOWEN & IBS & 7.0 & 13 & 45.9 & 19.6 & 13 & 31.5 & 18.2 & 31.4 & 407.8 \\
\hline LU & SMOKING & 3.0 & 25 & 7.8 & 1.5 & 25 & 3 & 2.6 & 61.5 & $1,538.5$ \\
\hline MELNICK & $\begin{array}{l}\text { TEST } \\
\text { ANXIETY }\end{array}$ & 3.0 & 9 & 181.67 & 22.23 & 9 & 148.6 & 17.12 & 18.2 & 164.0 \\
\hline MUTKE & $\begin{array}{l}\text { READING } \\
\text { COM }\end{array}$ & 5.0 & 94 & 65 & & 94 & 78 & & -20.0 & $-1,880.0$ \\
\hline PATTERSON & PAIN & 1.0 & 10 & 1.33 & 0.4 & 10 & 1.29 & 0.49 & 3.0 & 30.1 \\
\hline PATTERSON & PAIN & 1.0 & 11 & 48.33 & 26.23 & 11 & 38.33 & 28.63 & 20.7 & 227.6 \\
\hline RASKIN & $\begin{array}{l}\text { HYPERTENSI } \\
\text { ON }\end{array}$ & 4.0 & 8 & 151.3 & 16.7 & 8 & 146.9 & 22.7 & 2.9 & 23.3 \\
\hline RIEGEL & SMOKING & 4.0 & 29 & 100 & & 29 & 37.9 & & 62.1 & $1,800.9$ \\
\hline TAN & PAIN & 4.0 & 9 & 25.22 & 3.83 & 9 & 16.78 & 7.29 & 33.5 & 301.2 \\
\hline UNTAS & ANXIETY & 1.0 & 29 & 8.17 & 3.6 & 29 & 6.59 & 3.8 & 19.3 & 560.8 \\
\hline WALTERS & DISTRESS & 13.0 & 1 & 16 & & 1 & 7 & & 56.3 & 56.3 \\
\hline \multirow[t]{2}{*}{ WERNER } & CHILDBIRTH & 3.0 & 485 & 60.5 & 19.9 & 485 & 42.9 & 23.5 & 29.1 & $14,109.1$ \\
\hline & & 5.7 & 1,479 & & \multicolumn{5}{|c|}{ Corrected success rate average: $39.8 \%$} & $59,013.3$ \\
\hline
\end{tabular}

Sheet 2-HYP studies included

Abramowitz, E. G., Barak, Y., Ben-Avi, I., \& Knobler, H. Y. (2008). Hypnotherapy in the treatment of chronic combat-related PTSD patients suffering from insomnia: A randomized, zolpidem-controlled clinical trial. International Journal of Clinical and Experimental Hypnosis, 56(3), 270-280.

Alladin, A., \& Alibhai, A. (2007). Cognitive hypnotherapy for depression: An empirical investigation. International Journal of Clinical and Experimental Hypnosis, 55(2), 147-166.

Anbar, R. D., \& Savedoff, A. D. (2005). Treatment of binge eating with automatic word processing and self-hypnosis: A case report. American Journal of Clinical Hypnosis, 48(2-3), 191-198.

Andreychuk, T., \& Skriver, C. (1975). Hypnosis and biofeedback in the treatment of migraine headache. International Journal of Clinical and Experimental Hypnosis, 23(3), 172-183.

Askay, S. W., Patterson, D. R., Jensen, M. P., \& Sharar, S. R. (2007). A randomized controlled trial of hypnosis for burn wound care. Rehabilitation Psychology, 52(3), 247-253.

Barkley, R. A., Hastings, J. E., \& Jackson, T. L. (1977). The effects of rapid smoking and hypnosis in the treatment of smoking behavior. International Journal of Clinical and Experimental Hypnosis, 25(1), 7-17.

Bolocofsky, D. N., Spinler, D., \& Coulthard-Morris, L. (1985). Effectiveness of hypnosis as an adjunct to behavioral weight management. Journal of Clinical Psychology J. Clin. Psychol., 41(1), 35-41.

Boutin, G. E. (1978). Treatment of Test Anxiety by Rational Stage Directed Hypnotherapy: A case study. American Journal of Clinical Hypnosis, 21(1), 52-57.

Carmody, T., Duncan, C., Simon, J., Solkowitz, S., Huggins, J., Lee, S., \& Delucchi, K. (2008). Hypnosis for smoking cessation: A randomized trial. Nicotine \& Tobacco Research, 10(5), 811-818.

Cohen, Ha, Barzilai, A., \& Lahat, E. (1999). Hypnotherapy: An effective treatment modality for trichotillomania. Acta Paediatrica, 88(4), 407-410.

Deiker, T. E., \& Pollock, D. H. (1975). Integration of Hypnotic and Systematic Desensitization Techniques in the Treatment of Phobias: A case report. American Journal of Clinical Hypnosis, 17(3), 170-174. 
Dickson-Spillmann, M., Haug, S., \& Schaub, M. P. (2013). Group hypnosis vs. relaxation for smoking cessation in adults: A cluster-randomised controlled trial. BMC Public Health, 13(1), 1227.

Elkins, G. R., \& Rajab, M. H. (2004). Clinical Hypnosis For Smoking Cessation: Preliminary Results of a Three-Session Intervention.International Journal of Clinical and Experimental Hypnosis, 52(1), 73-81.

Elkins, G., Johnson, A., Fisher, W., Sliwinski, J., \& Keith, T. (2013). A pilot investigation of guided self-hypnosis in the treatment of hot flashes among postmenopausal women. International Journal of Clinical and Experimental Hypnosis, 61(3), 342-350.

Elkins, G. R., Ramsey, D., \& Yu, Y. (2014). Hypnotherapy for persistent genital arousal disorder: A case study. International Journal of Clinical and Experimental Hypnosis, 62(2), 215-223.

Enea, V., Dafinoiu, I., Opriş, D., \& David, D. (2014). Effects of hypnotic analgesia and virtual reality on the reduction of experimental pain among high and low hypnotizables. International Journal of Clinical and Experimental Hypnosis, 62(3), 360-377.

Galovski, T. E., \& Blanchard, E. B. (2002). Hypnotherapy and refractory irritable bowel syndrome: A single case study. American Journal of Clinical Hypnosis, 45(1), 31-37.

Galovski, T. E, \& Edward B B. (1998). The treatment of irritable bowel syndrome with hypnotherapy. Applied Psychophysiology and Biofeedback, 23(4), 219-232.

Gay, M. (2007). Effectiveness of hypnosis in reducing mild essential hypertension: A one-year follow-up. International Journal of Clinical and Experimental Hypnosis, 55(1), 67-83.

Goodin, B. R., Quinn, N. B., Kronfli, T., King, C. D., Page, G. G., Haythornthwaite, J. A., . . Mcguire, L. (2012). Experimental Pain ratings and reactivity of cortisol and soluble tumor necrosis factor- $\alpha$ receptor II following a trial of hypnosis: Results of a randomized controlled pilot study. Pain Med Pain Medicine, 13(1), 29-44.

Gow, M. A. (2006). Hypnosis with a blind 55-year-old female with dental phobia requiring periodontal treatment and extraction. Contemporary Hypnosis Contemp. Hypnosis, 23(2), 83-91.

Grøndahl, J. R., \& Rosvold, E. O. (2008). Hypnosis as a treatment of chronic widespread pain in general practice: A randomized controlled pilot trial. BMC Musculoskeletal Disorders BMC Musculoskelet Disord, 9(1), 124.

Hawkins, P. J., Liossi, C., Ewart, B. W., Hatira, P., Kosmidis, V. H., \& Varvutsi, M. (1995). Hypnotherapy for control of anticipatory nausea and vomiting in children with cancer: Preliminary findings. Psycho-Oncology, 4, 101-106.

Hawkins, P., \& Polemikos, N. (2002). Hypnosis treatment of sleeping problems in children experiencing loss. Contemporary Hypnosis Contemp. Hypnosis, 19(1), 18-24.

Horton-Hausknecht, J. R., Mitzdorf, U., \& Melchart, D. (2000). The effect of hypnosis therapy on the symptoms and disease activity in Rheumatoid Arthritis. Psychology \& Health, 14(6), 1089-1104.

Jensen, M. P., Barber, J., Hanley, M. A., Engel, J. M., Romano, J. M., Cardenas, D. D., . . Patterson, D. R. (2008). Long-term outcome of hypnotic-analgesia treatment for chronic pain in persons with disabilities. International Journal of Clinical and Experimental Hypnosis, 56(2), 156-169.

Jensen, M. P., Barber, J., Romano, J. M., Molton, I. R., Raichle, K. A., Osborne, T. L., . . Patterson, D. R. (2009). A comparison of self-hypnosis versus progressive muscle relaxation in patients with multiple sclerosis and chronic pain. International Journal of Clinical and Experimental Hypnosis, 57(2), 198-221.

Jensen, M. P., Barber, J., Romano, J. M., Hanley, M. A., Raichle, K. A., Molton, I. R., . . Patterson, D. R. (2009). Effects of self-hypnosis training and EMG biofeedback relaxation training on chronic pain in persons with spinal-cord injury. International Journal of Clinical and Experimental Hypnosis, 57(3), 139-268.

Johnson, L. S., Johnson, D. L., Olson, M. R., \& Newman, J. P. (1981). The uses of hypnotherapy with learning disabled children. Journal of Clinical Psychology J. Clin. Psychol., 37, 291-299.

Lindfors, P., Ljótsson, B., Bjornsson, E., Abrahamsson, H., \& Simrén, M. (2012). Patient satisfaction after gut-directed hypnotherapy in irritable bowel syndrome. Neurogastroenterology \& Motility, 25(2), 169-186.

Liossi, C., \& Hatira, P. (1999). Clinical hypnosis versus cognitive behavioral training for pain management with pediatric cancer patients undergoing bone marrow aspirations. International Journal of Clinical and Experimental Hypnosis, 47(2), 104-116.

Lowén, M. B. O., Mayer, E. A., Sjöberg, M., Tillisch, K., Naliboff, B., Labus, J., Lundberg, P., Ström, M., Engström, M., \& Walter, S. A. (2013). Effect of Hypnotherapy and Educational Intervention on Brain Response to Visceral Stimulus in the Irritable Bowel Syndrome. Alimentary Pharmacology \& Therapeutics Aliment Pharmacol Ther., 37(12), 1184-1197.

Lu, D. P., Lu, G. P., \& Kleinman, L. (2001). Acupuncture and clinical hypnosis for facial and head and neck pain: A single crossover comparison. American Journal of Clinical Hypnosis, 44(2), 141-148. 
Melnick, J., \& Russell, R. W. (1976). Hypnosis versus systematic desensitization in the treatment of test anxiety. Journal of Counseling Psychology, 23(4), 272-280.

Mutke, P. H. (1967). Increased reading comprehension through hypnosis. American Journal of Clinical Hypnosis, 9(4), $262-266$.

Patterson, D. (2006). Immersive virtual reality induced hypnosis for burn pain. PsycEXTRA Dataset, 54(2), 130-142.

Patterson, D. R., Jensen, M. P., Wiechman, S. A., \& Sharar, S. R. (2010). Virtual reality hypnosis for pain associated with recovery from physical trauma. International Journal of Clinical and Experimental Hypnosis, 58(3), 288-300.

Raskin, R., Raps, C., Luskin, F., Carlson, R., \& Cristal, R. (1999). Pilot study of the effect of self-hypnosis on the medical management of essential hypertension. Stress Medicine Stress Med., 15(4), 243-247.

Riegel, B. (2013). Hypnosis for smoking cessation: Group and individual treatment-A free choice study. International Journal of Clinical and Experimental Hypnosis, 61(2), 146-161.

Stradling, J., Roberts, D., Wilson, A., \& Lovelock, F. (1998). Controlled trial of hypnotherapy for weight loss in patients with obstructive sleep apnoea. Int J Obes Relat Metab Disord International Journal of Obesity, 22(3), 278-281.

Tan, G., Fukui, T., Jensen, M. P., \& Thornby, J. (2010). Hpnosis in the treatment of veterans with chronic low-back pain. PsycEXTRA Dataset, 58(1), 53-68.

Untas, A., Chauveau, P., Dupré-Goudable, C., Kolko, A., Lakdja, F., \& Cazenave, N. (2013). The effects of hypnosis on anxiety, depression, fatigue, and sleepiness in people undergoing hemodialysis: A clinical report. International Journal of Clinical and Experimental Hypnosis, 61(4), 475-483.

Walters, V. J., \& Oakley, D. A. (2002). Hypnosis in post-abortion distress: An experimental case study.Contemporary Hypnosis Contemp. Hypnosis,19(2), 85-99.

Werner, A., Uldbjerg, N., Zachariae, R., Wu, C. S., \& Nohr, E. A. (2013). Antenatal hypnosis training and childbirth experience: A randomized controlled trial. Birth, 40(4), 272-280. 